\title{
QUATERNIONIC REDUCTION AND EINSTEIN MANIFOLDS
}

\author{
CHARLES P. BOYER KRZYSZTOF GALICKI BENJAMIN M. MANN
}

\section{INTRODUCTION}

A quaternionic Kähler manifold $(M, g)$ is a Riemannian manifold of dimension $4 n$, where $n>1$, whose reduced holonomy group is a subgroup of $S p(n) \cdot S p(1)$. One extends this definition to the case when $n=1$ by saying a 4 dimensional quaternionic Kähler manifold is one that is both self-dual and Einstein. Ever since this type of geometry was introduced by Ishihara [I1] there has been a continuous effort to study and classify these spaces. Wolf [Wo] classified all compact, homogeneous, and symmetric quaternionic Kähler manifolds. This classification was extended to the non-compact case by Alekseevskii [A]. As the group $S p(n) \cdot S p(1)$ appears in Berger's [Ber] classification theorem as the possible holonomy group of a locally irreducible, non-symmetric Riemannian space, this naturally raises the question of the existence of nonsymmetric space examples. [A] and [G1] gave examples of non-compact manifolds with quaternionic Kähler metrics with negative scalar curvature which are not locally symmetric. If $(M, g)$ is compact with positive scalar curvature the classification problem has not been answered in general. There are, however, some classification results in dimension four $[\mathrm{H}]$ and eight [PoSal], where the only compact quaternionic Kähler manifolds of positive scalar curvature are, in fact, the symmetric Wolf spaces.

Many of the recent developments mentioned above were possible due to the introduction of various associated fibrations. First, Salamon [Sal1] constructed

During the preparation of this work all three authors were supported by NSF grants. 
a natural fibration

$$
\mathcal{Z}(M) \rightarrow M
$$

over any quaternionic Kähler manifold $M$, where the total space, $\mathcal{Z}(M)$, is called the twistor space of $M . \mathcal{Z}(M)$ is a complex manifold whose holomorphic structure encodes all the information about the Riemannian geometry of $M$. Moreover, if $M$ is compact with positive scalar curvature, then $\mathcal{Z}(M)$ is a Kähler-Einstein manifold. Salamon's construction was later inverted by Pedersen and Poon [PePo2] and by. LeBrun [L]. They show how a quaternionic Kähler geometry can be reconstructed from holomorphic data on a complex manifold with some additional structure. These results, which give a $4 n$ dimensional generalization of the twistor correspondence introduced by Penrose $[\mathrm{Pe}, \mathrm{AHS}]$ for self-dual 4-manifolds, make it possible to formulate many problems concerning the Riemannian geometry of the quaternionic Kähler manifolds in the language of the holomorphic geometry on the corresponding twistor spaces.

Next, Swann [Sw1, Sw2] introduced another fibration over any quaternionic Kähler manifold $M$ by defining an associated quaternionic bundle of $M$

$$
\mathcal{U}(M)=M \times_{S p(n) \cdot S p(1)}\left(\mathbb{H}^{*} /(\mathbb{Z} / 2)\right) \rightarrow M
$$

where the total space $\mathcal{U}(M)$ is a (pseudo)-hyperkähler manifold with some additional properties. It admits a certain isometric $G=S p(1)$, or $G=S O(3)$, action rotating the hyperkähler structure. This is locally equivalent to the requirement that $\mathcal{U}(M)$ has a hyperkähler potential function; i.e., a function which is simultaneously a Kähler potential with respect to every complex structure on the 2-sphere of complex structures. Yet another way to characterize $\mathcal{U}(M)$, which is locally equivalent to the two ways above, is to say that it admits a non-zero scalar curvature (pseudo)-quaternionic Kähler metric in the same quaternionic class. Swann demonstrates how to invert his bundle construction; that is, how to obtain a quaternionic Kähler manifold from a given hyperkähler space with these properties. In much the same way that the holomorphic data on $\mathcal{Z}(M)$ encodes all the information about the Riemannian geometry of $M$, the hyperkähler geometry in the associated bundle 
$\mathcal{U}(M)$ carries all the information about the quaternionic Kähler geometry of $M$. In some sense Swann's bundle is even simpler and more fundamental than the twistor space as $\mathcal{U}(M)$ fibers over both $M$ and $\mathcal{Z}(M)$ with fibers $\mathbb{H}^{*} /(\mathbb{Z} / 2)$ and $\mathbb{C}^{*} /(\mathbb{Z} / 2)$ respectively. The manifold $M$ and its twistor space $\mathcal{Z}(M)$ are thus obtained in a very natural way as $S p(1)$ and $S^{1}$ quotients of some hypersurface in $\mathcal{U}(M)$ given by the level sets of the hyperkähler potential function $\nu$. This construction led Swann to observe that nilpotent adjoint orbits of the complexification of a compact semisimple Lie group $L$, with the hyperkähler structure introduced by Kronheimer [Kr3], fiber this way over quaternionic Kähler spaces. Let us remark that the associated quaternionic bundle is a mathematical model for the scalar couplings in 4-dimensional $N=2$ conformal supergravity theory [G3].

In this paper we investigate another fibration over quaternionic Kähler base and its relations to both Swann's and Salamon's constructions. We begin in $\S 1$ with a hyperkähler manifold $M$ with an isometric $G$ action which permutes the hyperkähler structure, where $G$ is either $S p(1)$ or $S O(3)$. For any such action we define functions $\nu: M \rightarrow \mathbb{R}$ and $\phi: M \rightarrow S^{4} H$, where $H$ is the standard complex representation of $S p(1)$. The level sets of $\nu$ are $G$-invariant hypersurfaces in $M$ and, assuming that $G$ acts freely, we can introduce the quotient $M^{\prime}=\nu^{-1}(c) / G$. We show that the vanishing of the obstruction $\phi$ is both necessary and sufficient for $M^{\prime}$ to be a quaternionic Kähler manifold. It is easy to see that the vanishing of $\phi$ is equivalent to the condition Swann assumes about the $S p(1)$ action on a hyperkähler manifold which is locally a model for his associated bundle, and in this case our $M$ must be locally homothetic to his $\mathcal{U}\left(M^{\prime}\right)$. What is new here is that, in this more general situation, the vanishing of $\phi$ is also a necessary condition for $M^{\prime}$ to be quaternionic Kähler. We close Section 1 with various corollaries of our obstruction theory. For example, under the assumption that $\phi$ vanishes, we show that the Kähler quotient of $M$ by any $U(1) \subset G$ is Kähler-Einstein.

In Section 2 we turn our attention to the level sets of the function $\nu$. As they are hypersurfaces in a hyperkähler manifold they carry a natural almost contact metric 3-structure. When the obstruction $\phi$ vanishes $\nu$ becomes a 
hyperkähler potential function on $M$. We then show that the hypersurface $S_{c}=\nu^{-1}(c)$ is a 3-Sasakian manifold with the 3-Sasakian structure defined by the vector fields of the $G$ action. Moreover, when $G$ acts freely on $S_{c}$ then the level sets have a structure of a fibered Riemannian space and the Riemannian submersion $S_{c} \rightarrow M^{\prime}$ is the 3-Sasakian submersion. In particular, the level sets of the hyperkähler potential function in Swann's associated bundle are all Einstein manifolds and are compact and of positive scalar curvature whenever $M^{\prime}$ is compact and of positive scalar curvature.

Ishihara [I2] showed that when the distribution given by the Killing vector fields of a 3-Sasakian structure is regular then the manifold fibers naturally over a quaternionic Kähler manifold. Konishi [Kon] also showed that there is always a 3-Sasakian submersion over an arbitrary quaternionic Kähler manifold of positive scalar curvature. Our analysis shows that these two results are closely related to the work of Swann. More precisely, we prove

Theorem A. Let $G$ be $S p(1)$ or $S O(3)$ and let $M$ be a hyperkähler manifold admitting a free action of $G$ by isometries permuting the complex structures. Let $\mathcal{Z}(M)$ denote the twistor space of $M$, and suppose that the obstruction $\tilde{\phi} \in$ $H^{0}\left(\mathcal{Z}(M), \Lambda^{(1,0)}(4)\right)$ described in Section 3 vanishes. Furthermore, suppose the action of $G$ extends to a homothetic $F$ action on $M$, where $F$ is $\mathbb{H}^{*}$ or $\mathbb{H}^{*} /(\mathbb{Z} / 2)$ respectively. Then there is a diagram of locally trivial fibrations

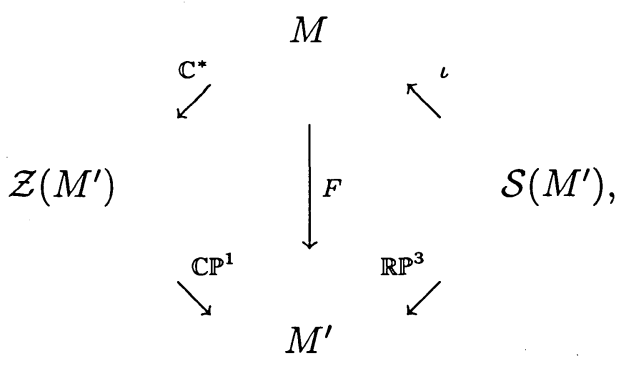

where all maps except $\iota$ are projections of the fibrations with fiber as indicated, $M^{\prime}$ is a quaternionic Kähler manifold of positive scalar curvature, $\mathcal{Z}\left(M^{\prime}\right)$ is its twistor space which is Kähler-Einstein of positive scalar curvature, $\mathcal{S}\left(M^{\prime}\right)$ is a fibered Riemannian Einstein manifold with 3-Sasakian structure and a second non-isometric Einstein metric, and $\iota$ is an embedding. Moreover, $M$ 
is homothetic to the associated quaternionic bundle $\mathcal{U}\left(M^{\prime}\right)$ of Swann.

Conversely, if $M^{\prime}$ is a quaternionic Kähler manifold of positive scalar curvature, then the diagram above holds with the stated properties, and the obstruction $\tilde{\phi}$ vanishes. Furthermore, if $M^{\prime}$ is complete, then $M^{\prime}, \mathcal{Z}\left(M^{\prime}\right)$, and $\mathcal{S}\left(M^{\prime}\right)$ are all compact.

In the case that the obstruction $\tilde{\phi}$ does not vanish, the manifold $M^{\prime}$ is not quaternionic Kähler, $\mathcal{S}\left(M^{\prime}\right)$ is an almost contact metric 3-structure, but not 3Sasakian, and $\mathcal{Z}\left(M^{\prime}\right)$ is a Kähler manifold, but without a fibering by 2 -spheres, and is not Kähler-Einstein. It is quite remarkable that all four geometries appearing in 0.3 are Einstein. As $\mathcal{S}\left(M^{\prime}\right)$ fibers naturally over $\mathcal{Z}\left(M^{\prime}\right)$ we have the following diagram:

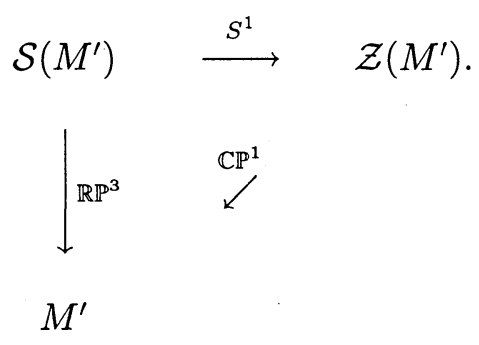

It is the generalization of the relation between the Hopf fibrations of $S^{4 k+3}$ over $\mathbb{H} \mathbb{P}^{k}$ and $\mathbb{C P}^{2 k+1}$ respectively, where $\mathbb{C P}^{2 k+1}$ fibers over $\mathbb{H} \mathbb{P}^{k}$ as its twistor space. Notice that the fibers themselves form the Hopf fibration $S^{1} \rightarrow \mathbb{R P} \mathbb{P}^{3} \rightarrow \mathbb{C P}^{1}$. The diagram 0.4 is well-known in the case when $M^{\prime}$ is a quaternionic Kähler manifold of positive scalar curvature [Bes]. The manifold $\mathcal{S}\left(M^{\prime}\right)$ admits two non-isometric Einstein metrics. It follows from Theorem A that $\mathcal{S}\left(M^{\prime}\right)$ is, in fact, a 3-Sasakian manifold with respect to one of these metrics.

One can generalize 0.4 to the case of "orbifold" fibrations, where $M^{\prime}$ is a quaternionic Kähler orbifold of positive scalar curvature. This can be seen as follows: If $G$ acts only locally freely on $S_{c}$ then, using theorem 2.1 , we obtain a 3-Sasakian structure on it but now, as the distribution defined by the vector fields of the $G$ action is not regular, $S_{c}$ no longer fibers over a quaternionic Kähler manifold. The manifold $S_{c}$ can be a compact 3-Sasakian space and the quotient $S_{c} / G$ yields a compact quaternionic Kähler orbifold with positive 
scalar curvature. We call such a distribution weakly regular (see also [Th]). In this case the projection map of $\mathcal{S}\left(M^{\prime}\right)$ onto the twistor space $\mathcal{Z}\left(M^{\prime}\right)$ has the structure of a Seifert fibration [OW].

Hence, we get an interesting generalization of Theorem A involving orbifolds:

Theorem B. Let $G$ be either. $S p(1)$ or $S O(3)$ and let $M$ be a hyperkähler manifold admitting a locally free (but not free) isometric action of $G$ permuting the complex structures. Let $\mathcal{Z}(M)$ denote the twistor space of $M$, and suppose that the obstruction $\tilde{\phi} \in H^{0}\left(\mathcal{Z}(M), \Lambda^{(1,0)}(4)\right)$ described in Section 3 vanishes. If the action of $G$ extends to a homothetic $F$ action on $M$, where $F$ is $\mathbb{H}^{*}$ or $\mathbb{H}^{*} /(\mathbb{Z} / 2)$ respectively, then there is a diagram

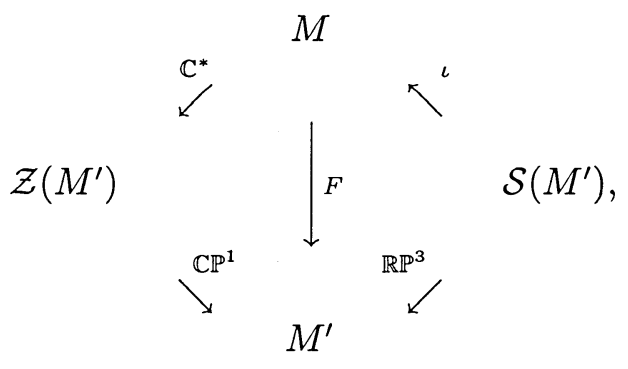

where all maps except $\iota$ and $\mathcal{Z}\left(M^{\prime}\right) \stackrel{\mathbb{C P}^{1}}{\longrightarrow} M^{\prime}$ are quotient maps indicated, $M^{\prime}$ is a quaternionic Kähler orbifold of positive scalar curvature, $\mathcal{Z}\left(M^{\prime}\right)$ is a complex contact orbifold which is Kähler-Einstein of positive scalar curvature, $\mathcal{S}\left(M^{\prime}\right)$ is an Einstein manifold with 3-Sasakian structure and a second non-isometric Einstein metric, $\iota$ is an embedding, and $\mathcal{Z}\left(M^{\prime}\right) \stackrel{\mathbb{C P}^{1}}{\longrightarrow} M^{\prime}$ is a twistor fibration away from the orbifold singularities of $M^{\prime}$. Moreover, $M$ is homothetic to the associated quaternionic bundle $\mathcal{U}\left(M^{\prime}\right)$ of Swann away from the orbifold singularities of $M^{\prime}$. Furthermore, if $M^{\prime}$ is complete, then $M^{\prime}, \mathcal{Z}\left(M^{\prime}\right)$, and $\mathcal{S}\left(M^{\prime}\right)$ are all compact.

We emphasize here that although $M^{\prime}$ and $\mathcal{Z}\left(M^{\prime}\right)$ are Einstein orbifolds, $\mathcal{S}\left(M^{\prime}\right)$ is a smooth Einstein manifold. Theorem B allows for a construction of 3-Sasakian manifolds that are not fibered Riemannian spaces, thus providing us with examples of new Einstein manifolds in dimension $4 n+3$. 
Both theorems A and B have straightforward generalizations to the pseudoRiemannian category. In particular, if $M^{\prime}$ is a quaternionic Kähler manifold or orbifold (Riemannian) of dimension $4 n$ with negative scalar curvature, then $\mathcal{U}\left(M^{\prime}\right), \mathcal{S}\left(M^{\prime}\right), \mathcal{Z}\left(M^{\prime}\right)$ have signature $(4 n, 4),(4 n, 3)$, and $(4 n, 2)$ respectively.

In Section 3 we briefly describe the twistor space of hyperkähler and quaternionic Kähler manifolds, and then show that the obstructions described in Section 1 are represented by an element $\tilde{\phi}$ of $H^{0}\left(\mathcal{Z}(M), \Lambda^{(1,0)}(4)\right)$ on the twistor space $\mathcal{Z}(M)$ of the hyperkähler manifold $M$.

We conclude this paper in Section 4 by giving many examples of hyperkähler manifolds with an isometric $G$ action permuting the hyperkähler structure which illuminate various aspects of the theory developed in the earlier sections. When the obstruction $\tilde{\phi}$ vanishes we obtain many new examples by using the quaternionic Kähler quotient [G2, GL] and hyperkähler quotient [HKLR] methods. If $M$ admits a group of hyperkähler isometries $H$ commuting with the $G$ action then, under some additional assumptions given in Section 4, we can construct a new manifold $\hat{M}$ which is a quotient of the zero level set of the momentum map $\mu$ by $H$. This quotient is precisely the quaternionic Kähler reduction of $M^{\prime}$ by $H$. It follows from [Sw1, Sw2] that the following diagram commutes:

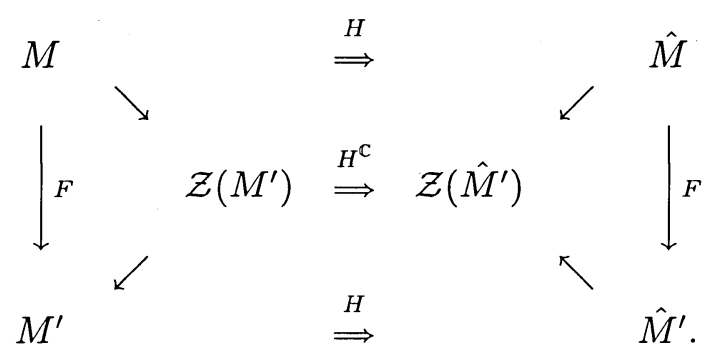

That is, $\hat{M}$ is locally homothetic to the associated bundle of $\hat{M}^{\prime}$. When $M=$ $\mathcal{U}\left(M^{\prime}\right)$ then $\hat{M}$ is homeomorphic to $\mathcal{U}\left(\hat{M}^{\prime}\right)$. The above diagram implicitly defines a reduction of the 3-Sasakian manifold $\mathcal{S}\left(M^{\prime}\right)$ by the action of $H$ to yield $\mathcal{S}\left(\hat{M}^{\prime}\right)$. When $M=\mathbb{H}^{k} \backslash\{0\}$ then we can take $H$ to be any subgroup of $S p(k)$ acting by matrix multiplication from the left and the hyperkähler quotient on $\mathbb{H}^{k} \backslash\{0\}$ becomes the quaternionic Kähler quotient on $\mathbb{H}^{k-1}$. 
For example, if $H=U(1)$ or $S p(1)$ we obtain diagram 0.3 when $M^{\prime}$ is a Grassmannian $G_{2, k}(\mathbb{C})$ or $G_{4, k}(\mathbb{R})$ respectively. In these cases, the $\mathcal{S}\left(M^{\prime}\right)$ are $\mathbb{R} \mathbb{P}^{3}$ bundles over quaternionic Kähler manifolds with the 3-Sasakian structure obtained by Konishi [Kon] and they are homogeneous Einstein manifolds. Using techniques described in this paper, together with the results of Swann [Sw1, Sw2], one can explicitly construct such fibrations for any compact symmetric Wolf space of positive scalar curvature. It also follows that the level sets of the hyperkähler potential function of the hyperkähler metric on the nilpotent adjoint orbit of the complexification of a compact semisimple Lie group $L$ carry a natural 3-Sasakian structure and, in particular, are Einstein manifolds.

In many ways the most interesting class of examples comes from considering the $S O(3)$ action on the associated bundle of the complex weighted projective space $\mathbb{C P}_{\frac{p+q}{2}}^{2}, \frac{q+s}{2}, \frac{p+s}{2}$, where $p, q, s$ are pairwise coprime positive integers and $(p+q+s)$ is odd. The orbifold $\mathbb{C P}_{\frac{p+q}{2}, \frac{q+s}{2}, \frac{p+s}{2}}^{2}$ has a singular locus consisting of three isolated points except when $p=q=1$ and has a self-dual Einstein metric of positive scalar curvature [GL]. The associated quaternionic bundle $\mathcal{U}\left(\mathbb{C P}_{\frac{p+q}{2}, \frac{q+s}{2}, \frac{p+s}{2}}^{2}\right)$ is an incomplete hyperkähler manifold with hyperkähler potential function $\nu$. The level sets of $\nu$ are smooth compact hypersurfaces with an induced 3-Sasakian structure. The space $S_{p, q, s}=\nu^{-1}(1 / 2)$ is, therefore, an example of a compact 3-Sasakian space for which the distribution defined by the vector fields of the $S O(3)$ action is not regular. However, it is almost (or weakly) regular [Th] and $S_{p, q, s} / S O(3)$ is the Riemannian orbifold $\mathbb{C P}_{\frac{p+q}{2}, \frac{q+s}{2}, \frac{p+s}{2}}^{2}$. In particular, $S_{p, q, s}$ is a compact Einstein 7-manifold of positive scalar curvature for any choice of such triple $(p, q, s)$. It is a quotient of $S U(3)$ by a free circle action. In the case $(p+q+s)$ is even $S_{p, q, s}$ has a locally free $S p(1)$ action and the projection $S_{p, q, s} / S p(1)=\mathbb{C P}_{p+q, p+s, q+s}$. We can also think of $S_{p, q, s}$ as a reduction of the standard 3-Sasakian structure on the 11-dimensional sphere by a circle action. The Einstein metric constructed on $S_{p, q, s}$ is non-homogeneous, and we believe these are new non-homogeneous compact Einstein manifolds of positive scalar curvature in odd dimension. Our theory allows construction of many other examples in dimension 7 as well as in a general dimension $4 k+3$. 
Even dimensional examples of compact non-homogeneous Einstein manifolds with positive cosmological constant were introduced first by Page [Pa] and later his construction was generalized by Bérard Bergery [BéBer]. Koiso and Sakane [KoiSa1, KoiSa2] constructed examples of non-homogeneous KählerEinstein metrics of positive scalar curvature on compact complex manifolds. Their method allows for the construction of such manifolds with arbitrary cohomogeneity. Tian and Yau [TY] have also constructed non-homogeneous Kähler-Einstein metrics on the del Pezzo surfaces $\mathbb{C P}^{2} \# k\left(-\mathbb{C P}^{2}\right)$ for $3 \leq k \leq$ 8. Wang and Ziller's showed that a circle bundle $P_{\alpha_{1}, \ldots, \alpha_{n}}$ over a product of Kähler-Einstein manifolds of positive scalar curvature $M_{1} \times \cdots \times M_{n}$ admits an Einstein metric of positive scalar curvature [WanZi1, WanZi2]. Now, if all $M_{i}$ 's are homogeneous so is $P$. But choosing non-homogeneous examples of Koiso and Sakane or Yau and Tian for some of the $M_{i}$ 's, one can construct non-homogeneous Einstein metrics on $P$ with positive Einstein constant and of arbitrary cohomogeneity. We believe our examples are different. They fiber over compact Kähler-Einstein orbifolds of positive scalar curvature but the circle action has the structure of a Seifert fibration. A detailed study of the geometry and topology of our orbifold examples is currently under investigation [BGM].

Recently Wang introduced another interesting construction of Einstein metrics on some principal bundles over products of quaternionic Kähler manifolds [Wan1]. In a sense it is a quaternionic analogue of the construction in [WanZi2]. Let $M_{i}, 1 \leq i \leq m$, be quaternionic Kähler manifolds of positive scalar curvature and $P_{i}$ the principal $S O(3)$ bundle over $M_{i}$. In our notation $P_{i}=\mathcal{S}\left(M_{i}\right)$. Let $F=[S O(3) \times \cdots \times S O(3)] / \triangle S O(3)$ ( $m$ factors $)$, and $\bar{M}$ be the fiber bundle associated to $P_{1} \times \cdots \times P_{m}$ with fiber $F$. Wang shows that $\bar{M}$ admits an Einstein metric with positive scalar curvature such that the projection $\pi: \bar{M} \rightarrow M_{1} \times \cdots \times M_{m}$ is a Riemannian submersion onto a product metric on $M_{1} \times \cdots \times M_{m}$ and such that the fibres are totally geodesic and isometric to $F$ equipped with a normal homogeneous metric. Wang observed that if all $M_{i}$ 's are homogeneous so is $\bar{M}$. Since all known examples of quaternionic Kähler manifolds of positive scalar curvature are homogeneous his construction yields only homogeneous Einstein metrics in the bundle. However, his 
theorem can be easily generalized using the results of this paper. Notice that, in our notation, $\bar{M}$ is just the quotient $\left[\mathcal{S}\left(M_{1}\right) \times \cdots \times \mathcal{S}\left(M_{m}\right)\right] / S O(3)$ where $S O(3)$ acts diagonally. We have

Theorem C. Let $S_{i}, 1 \leq i \leq m$, be compact 3-Sasakian manifolds of positive scalar curvature such that the distributions defined by the vector fields of the 3-Sasakian structures are weakly regular for all $i=1, \ldots, m$, i.e., the quotients $S_{i} / S O(3)$ are compact quaternionic Kähler orbifolds with positive scalar curvature. Define $S=\left(S_{1} \times \cdots \times S_{m}\right) / S O(3)$, where $S O(3)$ acts diagonally. Then $S$ is a compact Riemannian orbifold which admits an Einstein metric of positive scalar curvature at all regular points. Moreover, if the diagonal $S O(3)$ action is free on $S_{1} \times \cdots \times S_{m}$ then $S$ is a smooth compact Einstein manifold.

Using our non-homogeneous examples of weakly regular 3-Sasakian structures and the theorem above one can construct many new non-homogeneous Einstein metrics of positive scalar curvature in dimension $4 k+3, k>1$. There are similar "orbifold" generalizations of the Wang and Ziller's construction of Einstein metrics on torus bundles over products of Kähler-Einstein manifolds [BGM].

Our final example discusses applications of our theory to the hyperkähler structure and associated free $S p(1)$ action on instanton moduli space thus giving a companion theory to the one developed in [BoMa1].

We would like to thank Gerardo Hernandez for helpful conversations about 3-Sasakian manifolds. The second author would like to thank the Institute for Advanced Study for its support and hospitality. Some of this work was done during his stay there for the second term of 1990-1991.

\section{The Quaternionic Kähler Reduction Obstruction}

In this section we develop an obstruction theory to when a hyperkähler manifold with a locally free action of $S p(1)$ or $S O(3)$ via isometries has a quaternionic Kähler quotient. When our obstruction vanishes we obtain the assumptions of Swann, under which he proved such a quotient exists [Sw1, Sw2]. Conversely, we show that when our obstruction does not vanish, no 
such quotient can exist. We end this section with several other consequences implied by the vanishing of our obstruction.

Let $(M, g)$ be a hyperkähler manifold. Then $M$ comes equipped with complex structures $\left\{I^{a}\right\}_{a=1}^{3}$, which satisfy the quaternionic relations

$$
I^{a} I^{b}=-\delta^{a b}+\epsilon^{a b c} I^{c}
$$

The complex structures $\left\{I^{a}\right\}_{a=1}^{3}$ span a trivial three dimensional subbundle $\mathcal{I}$ of the endomorphism bundle $\operatorname{End}(T M)$. Dually, with respect to the hyperkähler metric $g$, there are three Kähler forms $\omega^{a}$ which span a trivial subbundle $\mathcal{G}$ of $\Lambda^{2} M$. Let $\operatorname{Aut}(\Omega) \subset \operatorname{Isom}(M, g)$ be a group of isometries acting on $(M, g)$ such that the four-form

$$
\Omega=\sum_{a=1}^{3} \omega^{a} \wedge \omega^{a}
$$

is invariant under the action of $\operatorname{Aut}(\Omega)$. Then there is a normal subgroup $H \subset \operatorname{Aut}(\Omega)$ which acts trivially on $\mathcal{G}$, i.e., $H$ leaves $\left\{\omega^{a}\right\}_{a=1,2,3}$ invariant. We call $H$ the group of hyperkähler isometries of $M$. Let $G \subset \operatorname{Aut}(\Omega)$ be either $S p(1)$ or $S O(3)$ and such that $G$ acts nontrivially on $\mathcal{G}$ by permuting the hyperkähler structure. For the remainder of this paper $G$ will denote either $S p(1)$ or $S O(3)$ and we assume that $G$ acts effectively on $M$.

Suppose that $G$ acts locally freely on a smooth manifold $M$. Let $\Gamma_{x}$ denote the isotropy subgroup at $x \in M . \Gamma_{x}$ is a finite subgroup of $G$ for all $x \in M$. Such an action gives rise to a weakly regular foliation $\mathcal{F}$ on $M$ whose leaf through $x$ is $G / \Gamma_{x}$. Since all the leaves are compact, the quotient space $M / \mathcal{F}$ has the structure of an orbifold or Satake manifold [Mo]. The union of the principal orbits is a dense open set which is a smooth manifold. There are two cases to consider.

CAse 1: The action of $G$ on $M$ is free. In this case the foliation is regular and the map $\pi: M \rightarrow M / G$ is a submersion, in fact, a principal $G$ bundle over $M / G$. We refer to this as the regular case.

CASE 2: The action of $G$ on $M$ is not free, but is locally free, and there is a dense set of points $\tilde{M} \subset M$ where the action is free. Over the dense open 
subset $\widetilde{M / \mathcal{F}} \subset M / \mathcal{F}$ of regular points, $\tilde{M}$ is a principal $G$ bundle over $\widetilde{M / \mathcal{F}}$. The fibres (singular fibres) over the singular points of $M / \mathcal{F}$ have the structure of $G / \Gamma_{x}, \Gamma_{x} \neq\{i d\}$. We refer to this case as the singular case. We treat the singular case by first working on the dense set of regular points and then adding back the singular fibres.

The complexified cotangent bundle $T_{\mathbb{C}}^{*} M$ of any $4 n$ dimensional quaternionic Kähler manifold $M$ can be written as $E \otimes H$ [Sal1] for locally defined vector bundles $E$ and $H$ of complex rank $2 n$ and 2 respectively. The fibres of $E$ and $H$ are the standard complex representations of $S p(n)$ and $S p(1)$ respectively. The quaternionic Kähler metric $g$ on $M$ can be given as an element $\omega_{E} \otimes$ $\omega_{H}$ in $\Lambda^{2} E \otimes \Lambda^{2} H \subset \Lambda^{2}(E \otimes H)$. When $M$ is hyperkähler the bundles $E$ and $H$ are globally defined and the form $\omega_{H}$ is the flat symplectic form $\epsilon$. Furthermore, there is an identification of the bundle $\mathcal{G}$ with the symmetric tensor product bundle $S^{2} H$. If $G$ acts freely on $M, M$ is the total space of a principal $G$ bundle. So there is a trivial three dimensional subbundle $\mathcal{V}$ of the tangent bundle $T M$ spanned by the fundamental vertical vector fields $\left\{\xi^{*}\right\}$ corresponding to $\xi \in \mathfrak{s p}(1)$. Of course, the fibres of $\mathcal{V}$ can be identified with the Lie algebra $\mathfrak{s p}(1)$, so we get the following moment map construction.

The Lie derivative gives a map $\mathcal{V} \otimes S^{2} H \rightarrow \mathfrak{s p}(1) \otimes S^{2} H$ defined by

$$
\left.\mathcal{L}_{\xi^{*}} \omega=d\left(\xi^{*}\right\rfloor \omega\right)=-[\xi, \omega]
$$

This gives rise to a moment map $\mu$ by identifying $\mathfrak{s p}(1)$ with $S^{2} H$ and decomposing

$$
S^{2} H \otimes S^{2} H \simeq 1 \oplus S^{2} H \oplus S^{4} H
$$

in irreducible components. The components of $\left.\xi^{*}\right\rfloor \omega$, with respect to each irreducible component in (1.4), give the factors of the moment map $\mu$. Since the right hand side of (1.3) lies in the second factor $S^{2} H$, the first and third factors of $\left.\xi^{*}\right\rfloor \omega$ are closed one-forms. Thus, assuming that $H^{1}(M, \mathbb{R})=0$, these closed forms are exact and we have

$$
\left.\xi^{*}\right\rfloor \omega=d \nu \oplus \eta \oplus d \phi
$$

with the following irreducible factors of $\mu$ : 
(1) $\nu: M \rightarrow \mathbb{R}$

(2) $\eta: T M \rightarrow \mathfrak{s p}(1) \simeq S^{2} H$

(3) $\phi: M \rightarrow S^{4} H$.

The first map $\nu$ is a $G$ invariant function. Thus, for any regular value $c \in \mathbb{R}$, $\nu^{-1}(c) / G$ is a Riemannian manifold in a natural way. We are interested in the geometric structures that this manifold can carry. In particular, under what conditions will $\nu^{-1}(c) / G$ be quaternionic Kähler?

Notice that (1.3) implies that

$$
d \eta=-2 \omega
$$

This, together with the simplicity of $\mathfrak{s p}(1)$, implies

Proposition 1.1. Let $(M, g)$ be a hyperkähler manifold on which there is an action of $G$ by isometries which act non-trivially on the bundle $\mathcal{G}$. Then the de Rham cohomology class of any Kähler form $\omega$ in $\mathcal{G}$ vanishes. In particular, any such $M$ is non-compact.

The $S p(1)$ (respectively, $S O(3)$ ) action on $M$ can be extended to an $\mathbb{H}^{*}$ (respectively, $\mathbb{H}^{*} / \mathbb{Z}_{2}$ ) action as follows. Since $\nu$ is an $G$ invariant function, the vector field $\operatorname{grad} \nu$ commutes with $\xi^{*}$ for any $\xi \in \mathfrak{s p}(1)$. Furthermore, dualizing equation 1.5 with respect to the metric $g$, we see that

$$
\operatorname{grad} \nu=\operatorname{tr} I \xi^{*}
$$

where $I$ is any complex structure in the two sphere of complex Kähler structures on $M$. It follows from (1.7) that $\operatorname{grad} \nu$ is nowhere vanishing on $M$ whenever $G$ acts locally freely. Now assume that $\operatorname{grad} \nu$ is complete. Then the Lie algebra $\mathfrak{s p}(1) \oplus \operatorname{sp}\{\operatorname{grad} \nu\}$ exponentiates to an $\mathbb{H}^{*}$ or $\mathbb{H}^{*} / \mathbb{Z}_{2}$ action on $M$. In this case we can identify $\nu^{-1}(c) / S p(1)$ with $M / \mathbb{H}^{*}$ or $\nu^{-1}(c) / S O(3)$ with $M /\left(\mathbb{H}^{*} / \mathbb{Z} / 2\right)$. 
Consider the diagram

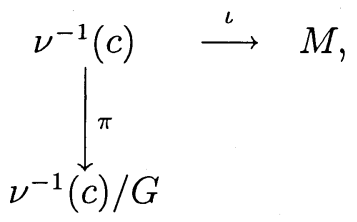

where the horizontal map is a Riemannian embedding and the vertical map is a principal Riemannian submersion. The pullback bundle $\iota^{*} T M$ on $\nu^{-1}(c)$ splits in terms of its vertical, normal, and horizontal components, respectively,

$$
\iota^{*} T M=\mathcal{V} \oplus \mathcal{N} \oplus \mathcal{H}
$$

We have bundles $\nu^{-1}(c) \times_{G} \iota^{*} \mathcal{I}$ and $\nu^{-1}(c) \times_{G} \iota^{*} \mathcal{G}$ on $M^{\prime}=\nu^{-1}(c) / G$.

We can define a bundle map

$$
\psi: \nu^{-1}(c) \times_{G} \iota^{*} \mathcal{G} \rightarrow \Lambda^{2} M^{\prime}
$$

as follows. On an open set $U_{\alpha}$ in $M^{\prime}$, choose a local trivialization $x \mapsto$ $\left([x], s_{\alpha}([x])\right)$ of the principal bundle $\nu^{-1}(c) \rightarrow M^{\prime}$. Then, with respect to this local trivialization, we can give a local section of the associated bundle $\nu^{-1}(c) \times_{G} \iota^{*} \mathcal{G}$ by setting $\omega_{\alpha}=\operatorname{ad}_{s_{\alpha}^{-1}} \omega$. Let $X^{\prime}$ and $Y^{\prime}$ be vector fields on $M^{\prime}$ and let $X$ and $Y$ be their unique horizontal lifts. Then we can define the map $\psi$ by $\psi\left(\omega_{\alpha}\right)\left(X^{\prime}, Y^{\prime}\right)=\omega_{\alpha}(X, Y)$. Equivalently, in terms of the almost complex structure, we have a map

$$
\psi^{\sharp}: \nu^{-1}(c) \times_{G} \iota^{*} \mathcal{I} \rightarrow \operatorname{End}\left(T M^{\prime}\right) .
$$

When does the image of $\psi$, or equivalently $\psi^{\sharp}$ define a quaternionic structure on $M^{\prime}$ ? A necessary condition is that $\psi$ or $\psi^{\sharp}$ be injective. We have

Lemma 1.2. The image of $\psi^{\sharp}$ defines an almost quaternionic structure on $M^{\prime}$ if and only if every complex structure $I \in \mathcal{I}$ restricted to $\nu^{-1}(c)$ leaves the horizontal subbundle $\mathcal{H}$ invariant.

Proof. If the horizontal subspace $\mathcal{H}_{x}$ for any $x \in \nu^{-1}(c)$ is invariant under any complex structure $I$, then the restriction $J$ of $I$ to $\mathcal{H}_{x}$ defines a complex structure on $\mathcal{H}_{x}$ for each $x \in \nu^{-1}(c)$. Thus, with respect to a local trivialization, $\psi^{\sharp}\left(I_{\alpha}\right)$ defines a quaternionic structure on $T_{[x]} M^{\prime} \simeq \mathcal{H}_{x}$ for each $[x] \in M^{\prime}$. 
Conversely, suppose that $I$ on $T_{x} M$ and $J$ defined as the horizontal component of the restriction of $I$ to $\mathcal{H}_{x}$ on $\mathcal{H}_{x}$ are both complex structures. Then with a choice of basis of $T_{x} M$ respecting the decomposition $T_{x} M=\mathcal{H}_{x} \oplus \mathcal{H}_{x}^{\perp}$, $I$ can be written in the block matrix form $\left(\begin{array}{ll}J & K \\ L & M\end{array}\right)$. Since $I^{2}=-1$ and $J^{2}=-1$ we have $K L=0$. But, as $I$ is an isometry, this implies that $I^{t}=-I$ and thus $L=-K^{t}$. Hence, $K K^{t}=0$ which implies that $K=L=0$. Thus, $\mathcal{H}_{x}$ is invariant.

In fact more is true:

Proposition 1.3. The structure induced by $\psi$ on $T M^{\prime}$ is an almost quaternionic structure if and only if it is quaternionic Kähler.

Proof. The if direction is obvious. The fact that the almost quaternionic structure implies $M^{\prime}$ is quaternionic Kähler follows from Lemma 1.2 and the proof of theorem 3.1 of Galicki and Lawson [GL].

Theorem 1.4. Let $(M, g, \mathcal{G})$ be a hyperkähler manifold with a free action of $G$ by isometries that induces the adjoint action on $\mathcal{G}$. Then the structure induced by $\psi$ on the quotient $M^{\prime}=\nu^{-1}(c) / G$ is a quaternionic Kähler structure if and only if $\phi$ is constant on $M$. Furthermore, if either condition is satisfied then we have

(1) $\mathcal{H} \oplus \mathcal{N}=$ ker $\eta$ and there is a choice of bases and of the constant in the moment map $\nu$ such that

(2) $\eta^{a}\left(X^{b}\right)=g\left(X^{a}, X^{b}\right)=2 \nu \delta^{a b}$.

Proof. The dual of (1.5) is

$$
I \xi^{*}=\operatorname{grad} \nu \oplus \operatorname{grad} \phi \oplus \eta^{\sharp} .
$$

Choose a basis for the Lie algebra $\mathfrak{s p}(1)$; for example, the basis $\left\{e_{a}\right\}_{a=1}^{3}$ corresponding to the imaginary quaternions so that $\left[e_{a}, e_{b}\right]=2 \epsilon^{a b c} e_{c}$. Let $\left\{X^{a}\right\}$ denote the corresponding basis for the fundamental vertical vector fields. Then, if $\phi$ is constant, writing (1.12) with respect to this basis gives

$$
I^{a} X^{b}=\delta^{a b} \operatorname{grad} \nu+\epsilon^{a b c}\left(\eta^{\sharp}\right)^{c} .
$$


Hence, we have

$$
I^{1} X^{1}=I^{2} X^{2}=I^{3} X^{3}=\operatorname{grad} \nu
$$

We show that the horizontal subbundle $\mathcal{H}$ is invariant under all the complex structures $I$. Then $\psi$ will induce a quaternionic Kähler structure on $M^{\prime}$ by Lemma 1.2 and Proposition 1.3. By orthogonality $\mathcal{H}$ is invariant under the complex structures if and only if $\mathcal{V} \oplus \mathcal{N}$ is. But (1.14) and (1.1) imply

$$
X^{a}=-I^{a} \operatorname{grad} \nu, \quad I^{a} X^{b}=\epsilon^{a b c} X^{c}+\delta^{a b} \operatorname{grad} \nu
$$

so $\mathcal{V} \oplus \mathcal{N}$ is invariant under the complex structures. Furthermore, it follows from (1.13) and (1.15) that $\left(\eta^{\sharp}\right)^{a}=X^{a}$, so that $\mathcal{H} \oplus \mathcal{N}=$ ker $\eta$.

Conversely, if the quotient is quaternionic Kähler, then $\mathcal{H}$ is invariant under the complex structures by Lemma 1.2. Hence, the righthand side of 1.12 can have no horizontal components. Clearly, this implies condition $\mathcal{H} \subset \operatorname{ker} \eta$. To show that $\phi$ must be constant on $M$ we prove a lemma. First notice that, restricted to the tangent bundle of the principal bundle $\nu^{-1}(c), \mathcal{H}$ defines a connection, and up to a change of basis, $\eta$ is its connection one-form.

Lemma 1.5. Under the hypothesis above, the Lie algebra of the holonomy group of the principal connection defined by $\mathcal{H}$ is $\mathfrak{s p}(1)$.

Proof. From (1.6) we have for any horizontal vector fields $X$ and $Y$ and any $\xi \in \mathfrak{s p}(1)$

$$
-2 \omega(X, Y)=d \eta(X, Y)=X \eta(Y)-Y \eta(X)-\eta([X, Y])=-\eta([X, Y]) .
$$

But the vertical component of $[X, Y]$ is essentially the curvature of the connection $\mathcal{H}$ of the principal $G$ bundle $\nu^{-1}(c)$. So, if the holonomy group were discrete, (1.16) would imply that any $\omega$ would be degenerate on $\mathcal{H}$. Similarly, if the restricted holonomy group were an $S^{1}$, then we could choose $\xi$ such that the nondegeneracy of $\omega$ were violated.

Lemma 1.5 says that any vertical vector can be written as the Lie bracket of horizontal vector fields. It follows that $\phi$ is annihilated by vertical vector fields 
as well as horizontal vector fields. Thus, we have shown that $\phi$ is constant on the level sets of $\nu$. This implies that (1.12) can be rewritten as

$$
I \xi^{*}=\left(1 \oplus f \oplus \frac{\eta(\operatorname{grad} \nu)}{\|\operatorname{grad} \nu\|^{2}}\right) \operatorname{grad} \nu \oplus\left(\eta^{\sharp}\right)^{v},
$$

where $f$ is an $S^{4} H$ valued function of $\nu$ alone and $\|\cdot\|$ denotes the norm with respect to the hyperkähler metric $g$. To show that $\phi$ is constant on $M$, we show that $f$ vanishes. The symmetric part of (1.17) gives the following set of equations

$$
\begin{array}{ll}
I^{1} X^{1}=\left(1+f^{11}\right) \operatorname{grad} \nu & I^{1} X^{2}+I^{2} X^{1}=2 f^{12} \operatorname{grad} \nu \\
I^{2} X^{2}=\left(1+f^{22}\right) \operatorname{grad} \nu & I^{3} X^{2}+I^{2} X^{3}=2 f^{23} \operatorname{grad} \nu \\
I^{3} X^{3}=\left(1+f^{33}\right) \operatorname{grad} \nu & I^{1} X^{3}+I^{3} X^{1}=2 f^{13} \operatorname{grad} \nu
\end{array}
$$

A straightforward calculation, using (1.1) and (1.18), together with the fact that $f$ is traceless, implies $f=0$ and, hence, that $\phi$ is constant on $M$.

Finally, to prove condition (2) we notice that from (1.14) we have $\left.g\left(X^{a}, X^{b}\right)=\omega^{a}\left(X^{b}, \operatorname{grad} \nu\right)=X^{b}\right\rfloor \omega^{a}(\operatorname{grad} \nu)=\delta^{a b} d \nu(\operatorname{grad} \nu)+\epsilon^{a b c} \eta^{c}(\operatorname{grad} \nu)$.

But the left hand side is symmetric in $a b$ so we get $\eta^{c}(\operatorname{grad} \nu)=0$ and

$$
\eta^{a}\left(X^{b}\right)=g\left(X^{a}, X^{b}\right)=\delta^{a b}\|\operatorname{grad} \nu\|^{2} .
$$

Let us set $u=\|\operatorname{grad} \nu\|^{2}$. We show first that $u$ depends only on $\nu$. From (1.16) we have,

$$
0=-2 \omega^{a}\left(X^{a}, X^{b}\right)=d \eta^{a}\left(X^{a}, X^{b}\right)=X^{a} \eta^{a}\left(X^{b}\right)-X^{b} \eta^{a}\left(X^{a}\right)-\eta^{a}\left(\left[X^{a}, X^{b}\right]\right)
$$

For $a \neq b$ this implies $X^{b} u=0$. Therefore $u$ is independent of the vertical directions. Similarly, the invariance of $\mathcal{H}$ under the $G$ action implies that $H u=0$ for any horizontal vector field $H$. Thus, $u$ is a function of $\nu$ only. Next, a similar computation using (1.19) gives

$$
\begin{aligned}
2\|\operatorname{grad} \nu\|^{2} \delta^{a b} & =2 g\left(X^{a}, X^{b}\right) \\
& =-2 \omega^{a}\left(\operatorname{grad} \nu, X^{b}\right) \\
& =(\operatorname{grad} \nu) \eta^{a}\left(X^{b}\right)-X^{b} \eta^{a}(\operatorname{grad} \nu)-\eta^{a}\left(\left[\operatorname{grad} \nu, X^{b}\right]\right)
\end{aligned}
$$


The second term on the right hand side vanishes by condition (1), and the third term vanishes by the $G$ invariance of $\nu$. Thus, we have the ODE

$$
(\operatorname{grad} \nu) u=2\|\operatorname{grad} \nu\|^{2} .
$$

This has a global solution $u=2 \nu+c$ where $c$ is a constant. By the standard uniqueness theorem, $u$ is uniquely determined by $c$. But, since $\nu$ was only defined up to a constant we can take $c=0$. This proves condition (2). Notice that with this choice of constant, we have

$$
\|\operatorname{grad} \nu\|^{2}=2 \nu .
$$

Remark 1.1. Conditions (1.14) are precisely the conditions assumed by Swann [Sw1, theorem 3.5.1] to obtain a quaternionic Kähler quotient. On the other hand, Theorem 1.4 shows these conditions are not only sufficient but also necessary conditions for $(M, g, \mathcal{G})$ to have a quaternionic Kähler quotient.

Proposition 1.6. Let $(M, g, \mathcal{G})$ be a hyperkähler manifold with a free action of $G$ by isometries that induce the adjoint action on $\mathcal{G}$ and such that the hypothesis of Theorem 1.4 is satisfied. Then

(1) $\mathcal{L}_{\operatorname{grad} \nu} \nu=2 \nu$,

(2) $\mathcal{L}_{\operatorname{grad} \nu} \omega^{a}=2 \omega^{a}$,

(3) $\operatorname{grad} \nu$ is a homothety of the metric $g$.

Proof. To prove (1) we notice that $\mathcal{L}_{\operatorname{grad} \nu} \nu=d \nu(\operatorname{grad} \nu)=\|\operatorname{grad} \nu\|^{2}=2 \nu$.

We show (2) by direct calculation. Assume $b \neq 1$. Then

$$
\begin{aligned}
\mathcal{L}_{\mathrm{grad} \nu} \omega^{b} & \left.=d\left(I^{1} X^{1}\right\rfloor \omega^{b}\right)=d\left(g\left(I^{b} I^{1} X^{1}, \cdot\right)\right) \\
& \left.=\epsilon^{b 1 c} d\left(g\left(I^{c} X^{1}, \cdot\right)\right)=\epsilon^{b 1 c} d\left(X^{1}\right\rfloor \omega^{c}\right) \\
& =\epsilon^{b 1 c} d\left(\delta^{1 c} d \nu+\epsilon^{c 1 d} \eta^{d}\right)=\epsilon^{b 1 c} \epsilon^{c 1 d} d \eta^{d} \\
& =-\epsilon^{b 1 c} \epsilon^{c 1 d} 2 \omega^{d}=2 \omega^{b} .
\end{aligned}
$$

When $b=1$ we repeat the same argument with $\operatorname{grad} \nu=I^{2} X^{2}$.

Finally (3) follows from the fact that $\left\{\omega^{a}\right\}_{a=1,2,3}$ are the Kähler forms and $\operatorname{grad} \nu$ is holomorphic with respect to any of the complex structures. 
Proposition 1.6 has several important corollaries which follow under the assumption of its hypothesis.

Corollary 1.7. $\left\{\operatorname{grad} \nu, X^{1}, X^{2}, X^{3}\right\}$ generates a local homothetic $\mathbb{H}^{*}$ action on $M$.

Corollary 1.8. The quaternionic Kähler metrics $g_{c}^{\prime}$ on $M^{\prime}=\nu^{-1}(c) / G$ obtained from different level sets of $\nu$ are homothetic.

The function $\nu$, in this case, is called the hyperkähler potential on $M$; i.e., it is a Kähler potential with respect to any complex structure $I \in \mathcal{I}$ [Sw1, Sw2]. Swann shows that such a $\nu$ completely determines the hyperkähler metric on $M$. In fact, with our normalization,

$$
\nabla^{2} \nu=g
$$

as one can easily see from (1.21). Moreover, the orbits of the $\mathbb{H}^{*}$ or $\mathbb{H}^{*} /(\mathbb{Z} / 2)$ action are totally geodesic and flat. He also shows that any hyperkähler manifold with a hyperkähler potential is locally homothetic to the associated quaternionic bundle of its quaternionic Kähler quotient or, in our notation,

Proposition 1.9. If $M$ satisfies the hypothesis of the Theorem 1.4 then it is locally homothetic to $\mathcal{U}\left(M^{\prime}\right)$ where $\mathcal{U}\left(M^{\prime}\right)$ is the associated quaternionic bundle of $M^{\prime}$. Moreover, $M \subset \mathcal{U}\left(M^{\prime}\right)$ is an open submanifold, and $\mathcal{U}\left(M^{\prime}\right)$ is its homothety completion.

Since Swann's associated quaternionic bundle $\mathcal{U}\left(M^{\prime}\right)$ is associated to the principal frame bundle on $M^{\prime}$ with group $S p(n) \cdot S p(1), \mathcal{U}\left(M^{\prime}\right)$ is naturally an $\mathbb{H}^{*} /(\mathbb{Z} / 2)$ bundle, and the obstruction to lifting the $\mathbb{Z} / 2$ is the MarchiafavaRomani class $\epsilon$ on $M^{\prime}$ [MR]. Furthermore, Salamon [Sal1] has shown that, if $M^{\prime}$ is a complete quaternionic Kähler manifold of positive scalar curvature, then $\epsilon$ vanishes if and only if $M^{\prime}=\mathbb{H} \mathbb{P}^{n}$. Thus, if we assume that our obstruction $d \phi$ vanishes and that $M^{\prime}$ is complete, then the only case where $G=S p(1)$ is when $M$ is an open submanifold of the quaternionic vector space $\mathbb{H}^{n+1}$ with its flat hyperkähler metric. This is not true when $M^{\prime}$ is a quaternionic Kähler orbifold. 
Another consequence of the equation (1.22) is that the metric $g$ on $\mathcal{U}\left(M^{\prime}\right)$ cannot be complete (see [Ya]).

When we fix a complex structure $I \in \mathcal{I}$ then $M$ is a Kähler manifold with Kähler form $\omega_{I}$ and a holomorphic circle action generated locally by $-I \operatorname{grad} \nu$. We can then consider an ordinary Kähler quotient of $M$ by this circle action.

Corollary 1.10. A Kähler quotient of $M$ by any circle subgroup $U(1) \subset G$ is Kähler-Einstein; that is, $Z=\nu^{-1}(c) / U(1)$ is a Kähler-Einstein manifold.

The Kähler-Einstein quotients were first studied by Futaki in the case when $M$ is compact and of positive scalar curvature [F]. He showed that if $M$ admits a holomorphic circle action by isometries such that the reduced space $\hat{M}$ is a manifold then the metric on $\hat{M}$ is Kähler-Einstein if and only if the norm of the vector field for the circle action is constant on the level sets of the momient map. In the more general case of an arbitrary Kähler-Einstein space this condition is not enough. This can be easily seen by the following example. One can consider an $S^{1}$ action on $\mathbb{C}^{k} \times \mathbb{C}^{m}$ where $S^{1}$ acts diagonally on $\mathbb{C}^{k}$ and trivially on $\mathbb{C}^{m}$. The vector field for this action is clearly constant on the level sets of the momentum map. The level sets are simply $S^{2 k-1} \times \mathbb{C}^{m}$ and the ordinary Kähler quotient yields $\mathbb{C} P^{k-1} \times \mathbb{C}^{m}$ with the product metric. While this is again a Kähler manifold it is not Einstein.

However, Pedersen and Poon [PePo1] observed that if one requires, in addition to the vector field being constant on each level set, that the quotient metrics coming from different level sets are homothetic then the quotient metric is not only Kähler but also Einstein. Both of the conditions are satisfied for any circle subgroup of $G$ acting on $M$ and, as a consequence, we get Corollary 1.10. In Section 3 we shall see that $Z=\mathcal{Z}\left(M^{\prime}\right)$ is, in fact, the twistor space of $M^{\prime}$.

In the next section we shall study the Riemannian geometry of the level sets of the hyperkähler potential function $\nu$. We will demonstrate that in the case of vanishing obstructions they carry a natural 3-Sasakian structure. In particular, the metric given by the inclusion map is Einstein. 


\section{Three-Sasakian Structure}

In this section we will see how 3-Sasakian structures arise naturally in the context discussed in $\S 1$. To begin we review some basic definitions and facts about Sasakian and 3-Sasakian structures (see for example [Ku, Sas1, Sas2, IK, I2, SasHa, Kon, Ka]). Let $S$ be a differentiable manifold with a type $(1,1)$ tensor field $\Phi$, a vector field $\xi \in \mathcal{X}(S)$, and a 1-form $\eta \in \Lambda T^{*} S$ such that

$$
\begin{gathered}
\Phi \circ \Phi(Y)=-Y+\eta(Y) \xi, \\
\Phi \xi=0, \\
\eta(\Phi Y)=0,
\end{gathered}
$$

and

$$
\eta(\xi)=1,
$$

for any vector $Y \in T S$. Then $(S, \Phi, \xi, \eta)$ is called an almost contact manifold. Given any such $S$ we can always choose a Riemannian metric $g$ with the following properties

$$
g(Y, \xi)=\eta(Y)
$$

and

$$
g(\Phi Y, \Phi Z)=g(Y, Z)-\eta(Y) \eta(Z),
$$

for any $Y, Z \in T S$. The metric $g$ is said to be associated to the almost contact structure and $(g, \Phi, \xi, \eta)$ is called an almost contact metric structure. An almost contact metric structure is a contact structure if, for any $Y, Z \in T S$,

$$
d \eta(Y, Z)=2 g(\Phi Y, Z) .
$$

Let us denote the Nijenhuis torsion tensor of $\Phi$ by $N_{\Phi}$, that is

$$
N_{\Phi}(Y, Z)=[\Phi Y, \Phi Z]+\Phi^{2}[Y, Z]-\Phi[Y, \Phi Z]-\Phi[\Phi Y, Z] .
$$

The almost contact structure $(\Phi, \xi, \eta)$ is normal [Sas1] if

$$
N_{\Phi}(Y, Z)=-d \eta(Y, Z) \otimes \xi .
$$


$(S, g, \Phi, \xi, \eta)$ is called a Sasakian manifold if $(g, \Phi, \xi, \eta)$ is a contact metric structure which is normal. In particular, it follows that the vector field $\xi$ is a Killing vector field on $S$.

Suppose now there are three distinct Sasakian structures $\left\{\Phi^{i}, \xi^{i}, \eta^{i}\right\}_{i=1,2,3}$ on $(S, g)$ such that

$$
g\left(\xi^{i}, \xi^{j}\right)=\delta^{i j}, \quad\left[\xi^{i}, \xi^{j}\right]=2 \epsilon^{i j k} \xi^{k}, \quad i, j, k=1,2,3 .
$$

Then $\left(g,\left\{\Phi^{i}, \xi^{i}, \eta^{i}\right\}_{i=1,2,3}\right)$ is called a 3 -Sasakian structure and $S$ is called a 3-Sasakian manifold. This structure implies that $S$ must have dimension $n=$ $4 k+3$. The distribution $\mathcal{F}$ spanned by $\left\{\xi^{i}\right\}_{i=1,2,3}$ is integrable and every integral manifold of $\mathcal{F}$ is totally geodesic in $S$ and of constant curvature 1 [IK]. Moreover, if the distribution $\mathcal{F}$ is regular then there is a natural projection $\pi: S \rightarrow M^{\prime}=S / \mathcal{F}$ where $M^{\prime}$ is the set of all maximal integral submanifolds of $\mathcal{F} .(S, g)$ is called a fibered Riemannian space with Sasakian 3-structure $\left\{\Phi^{i}, \xi^{i}, \eta^{i}\right\}$ and $\pi$ is a 3 -Sasakian submersion. It is known that any manifold with 3-Sasakian structure is Einstein $[\mathrm{Ka}]$ and that the base space $M^{\prime}$ of a 3-Sasakian submersion is a quaternionic Kähler manifold [I2]. Konishi [Kon] constructed a 3-Sasakian submersion over an arbitrary quaternionic Kähler manifold of positive scalar curvature.

We shall show that, under the assumptions of the Theorem 1.4, $\nu^{-1}(1 / 2)$ has a natural 3-Sasakian structure and the projection map $\pi$ of (1.8) is a 3-Sasakian submersion. Let $S=\nu^{-1}\left(\frac{1}{2}\right) \subset M$. Since, by definition, $S$ is $G$ invariant, the Killing vector fields on $M$,

$$
X^{i}=-I^{i} \operatorname{grad} \nu, \quad i=1,2,3
$$

can be considered as vector fields on $S$. Let $\tilde{g}$ be the metric on $S$ given by restriction of the hyperkähler metric $g$ on $M$ to the hypersurface $S$; i.e., $\tilde{g}=i^{*} g$, and let $p$ be the natural orthogonal projection $p: T_{m} M \rightarrow T_{m} S$, $m \in S$. We define

$$
\tilde{\eta}^{i}(Y)=\tilde{g}\left(Y, X^{i}\right), \quad Y \in T S, \quad i=1,2,3,
$$


and

$$
-\Phi^{i}(Y)=p \circ I^{i} Y=I^{i} Y-\tilde{\eta}^{i}(Y) \operatorname{grad} \nu, \quad Y \in T S, \quad i=1,2,3
$$

Theorem 2.1. The manifold $S$ is a fibered Riemaniann space with the 3Sasakian structure $\left(\tilde{g},\left\{X^{i}, \tilde{\eta}^{i}, \Phi^{i}\right\}\right)$ and $\pi: S \rightarrow M^{\prime}=S / G$ is a 3-Sasakian submersion. In fact, $S$ is a principal $G$ bundle over the quaternionic Kähler manifold $M^{\prime}$.

Proof. It is enough to show that $\left(\tilde{g}, X^{1}, \Phi^{1}, \tilde{\eta}^{1}\right)$ is a Sasakian structure because the conditions in (2.5) follow from Proposition 1.6. First we show that $\left(\tilde{g}, X^{1}, \Phi^{1}, \tilde{\eta}^{1}\right)$ defines a contact metric structure on $S$. Notice that $(1.12)$ and (1.13) imply that

$$
\left.\eta^{1}=X^{3}\right\rfloor \omega^{2}=g\left(I^{2} X^{3}, \cdot\right)=g\left(X^{1}, \cdot\right)
$$

In fact $\eta^{i}(Y)=g\left(Y, X^{i}\right), i=1,2,3$ for any $Y \in T M$. Thus, the $\tilde{\eta}^{i}$ defined on $S$ by (2.7) are pullbacks of the $\eta^{i}$ 's from $M$ to $S$ by the inclusion map; i.e, $\tilde{\eta}^{i}=i^{*} \eta^{i}$, for $i=1,2,3$.

We first check condition (2.3). As

$$
\begin{aligned}
-\tilde{g}\left(\Phi^{1} Y, Z\right) & =-g\left(\Phi^{1} Y, Z\right)=g\left(I^{1} Y-\eta^{1}(Y) \operatorname{grad} \nu, Z\right) \\
& =g\left(I^{1} Y, Z\right)-\eta^{1}(Y) g(\operatorname{grad} \nu, Z)=\omega^{1}(Y, Z)=i^{*} \omega^{1}(Y, Z)
\end{aligned}
$$

for all $Y, Z \in T S,(2.3)$ implies that $d \tilde{\eta}^{i}=-i^{*} 2 \omega^{i}$, for $i=1,2,3$. But this follows from (1.6) and the fact that pullbacks commute with the exterior differentiation. Equation (2.1d) follows from Theorem 1.4 and condition (2.1a)-(2.1c) follow by simple algebra:

$$
\begin{aligned}
-\tilde{\eta}^{1}\left(\Phi^{1} Y\right) & =-\tilde{g}\left(\Phi^{1} Y, X^{1}\right)=g\left(I^{1} Y-\eta^{1}(Y) \operatorname{grad} \nu, X^{1}\right) \\
& =g\left(I^{1} Y, X^{1}\right)-\eta^{1}(Y) g\left(\operatorname{grad} \nu, X^{1}\right)=-g\left(Y, I^{1} X^{1}\right) \\
& =-g(Y, \operatorname{grad} \nu)=0, \\
-\Phi^{1}\left(X^{1}\right) & =I^{1} X^{1}-\eta^{1}\left(X^{1}\right) \operatorname{grad} \nu=I^{1} X^{1}-\operatorname{grad} \nu=0, \\
\Phi^{1} \circ \Phi^{1}(Y) & =-I^{1}\left(\Phi^{1} Y\right)+\tilde{\eta}^{1}\left(\Phi^{1} Y\right) \operatorname{grad} \nu=I^{1}\left(I^{1} Y-\tilde{\eta}^{1}(Y) \operatorname{grad} \nu\right) \\
& =-Y+\tilde{\eta}^{1}(Y) X^{1}, \quad Y \in T S .
\end{aligned}
$$


Condition $(2.2 \mathrm{a})$ is true by definition and next we check $(2.2 \mathrm{~b})$ :

$$
\begin{aligned}
\tilde{g}\left(\Phi^{1} Y, \Phi^{1} Z\right)= & g\left(I^{1} Y-\eta^{1}(Y) \operatorname{grad} \nu, I^{1} Z-\eta^{1}(Z) \operatorname{grad} \nu\right) \\
= & g(Y, Z)+\eta^{1}(Y) \eta^{1}(Z)\|\operatorname{grad} \nu\|^{2} \\
& -\eta^{1}(Z) g\left(I^{1} Y, \operatorname{grad} \nu\right)-\eta^{1}(Y) g\left(I^{1} Z, \operatorname{grad} \nu\right) \\
= & g(Y, Z)-2 \nu \eta^{1}(Y) \eta^{1}(Z)-2 \eta^{1}(Y) \eta^{1}(Z) \\
= & g(Y, Z)-\eta^{1}(Y) \eta^{1}(Z)(2+2 \nu) \\
= & \tilde{g}(Y, Z)-\tilde{\eta}^{1}(Y) \tilde{\eta}^{1}(Z), \quad Y, Z \in T S .
\end{aligned}
$$

This completes the proof that $\left(\tilde{g}, \Phi^{1}, X^{1}, \tilde{\eta}^{1}\right)$ is the contact metric structure. We need to show that this structure is normal. This can be done again by an explicit calculation. However, the normality of the contact structure also follows from the fact that $S$ is a hypersurface in the Kähler manifold and the vector field $X^{1}$ is Killing [YK].

Notice that, since the metrics on $S_{c}=\nu^{-1}(c)$ are homothetic, a 3-Sasakian structure can be defined on an arbitrary level set.

Thus, Theorem 2.1 shows that there is a 3-Sasakian geometry naturally associated to any quaternionic Kähler manifold $M^{\prime}$ of positive scalar curvature. One can simply consider Swann's associated quaternionic bundle $\mathcal{U}\left(M^{\prime}\right)$ of $M^{\prime}$. This is a hyperkähler manifold with a hyperkähler potential function $\nu$ and an $S p(1)$ or $S O(3)$ action as in Theorem 1.4. The level sets of this function carry a 3-Sasakian structure, and they are either principal $S^{3}$ or $\mathbb{R} \mathbb{P}^{3}$ bundles over the base space $M^{\prime}$. Thus, we have an alternative proof of Konishi's result [Kon] mentioned previously. The relationship between the quaternionic Kähler geometry of $M^{\prime}$, the Kähler-Einstein geometry of the twistor space, the 3Sasakian geometry of the level sets of the hyperkähler potential function, and the hyperkähler geometry of the associated bundle $\mathcal{U}\left(M^{\prime}\right)$ are described by diagram (0.3). When $M$ is the flat space then the fiber of Swann's associated bundle is in fact $\mathbb{H}^{*}$ and the fiber of the 3-Sasakian submersion is $S^{3}$.

An interesting corollary of Theorem 2.1 and known results about 3-Sasakian manifolds is: 
Corollary 2.2. The manifold $S$ is an Einstein manifold of positive scalar curvature, and the fibres of $\pi$ are totally geodesic of constant curvature 1 . Hence, if $M^{\prime}$ is complete, both $M^{\prime}$ and $\mathcal{S}\left(M^{\prime}\right)$ are compact, and the fundamental group of $\mathcal{S}\left(M^{\prime}\right)$ is either $\{0\}$ or $\mathbb{Z} / 2$.

Proof. That a 3-Sasakian manifold is Einstein is well-known [Ka], and Kuo and Tachibana (cf. [IK]) showed that the fibres of a fibered Riemannian manifold with a 3-Sasakian structure are totally geodesic of constant curvature 1. Assuming that $S$ has positive scalar curvature, Myers' theorem says that $S\left(M^{\prime}\right)$ is compact with finite fundamental group. But $M^{\prime}$ is known to be simply connected [Sal1], so the last statement follows from the long exact sequence in homotopy. It remains to show that $S$ has positive scalar curvature. This follows from results in chapter 9 of Besse [Bes]. Since $S$ is Einstein with totally geodesic fibres, [Bes, 9.36, and 9.61], implies that $r=\lambda g$, where $r$ and $g$ are the Ricci tensor and metric of $S$ respectively. Thus, the scalar curvature $s$ of $S$ is $(4 n+3) \lambda$. Now for a Riemannian manifold of dimension $n$ with constant curvature $\kappa$, its scalar curvature is $n(n-1) \kappa$. So $\hat{s}=6$ where $\hat{s}$ is the scalar curvature of the fibres, and [Bes, 9.62a] gives $|A|^{2}=3 \lambda-6$, where $A$ is O'Neill's tensor. Thus, Lemma 1.5 implies that $\lambda>2$, so

$$
s=(4 n+3) \lambda>2(4 n+3)>0 \text {. }
$$

Another immediate corollary of Theorem 2.1 and results of Hitchin $[\mathrm{H}]$ and Poon and Salamon [PoSal] is:

Corollary 2.3. Let $S$ be a complete principal Riemannian fibration with 3Sasakian structure of positive scalar curvature. Then,

(1) if $S$ has dimension 7, then $S$ is either $S^{7}, \mathbb{R P}^{7}$, or $S U(3) / U(1)$.

(2) if $S$ has dimension 11 , then $S$ is either $S^{11}, \mathbb{R} \mathbb{P}^{11}, S U(4) / S(U(2) \times$ $U(1))$, or $G_{2} / S U(2)$.

In particular, every such fibered Riemannian manifold of dimension 7 or 11 with 3-Sasakian structure of positive scalar curvature is homogeneous.

One of the assumptions in Theorem 2.1 is that the $G$ action on $M$ is free. 
We do not need this assumption to show that the level sets of the hyperkähler potential have locally a 3 -Sasakian structure. For suppose that $G$ acts only locally freely on $M$. If the action is not free on $S_{c}$ then $S_{c}$ will not fiber over the quaternionic Kähler base. In other words the distribution defined by the vector fields of the $G$ action is not regular. Notice, however, that in this case $S_{c}$ still is homothetic to a 3-Sasakian manifold and the quotient $M^{\prime}=S_{c} / G$ is a quaternionic Kähler orbifold of positive scalar curvature. Furthermore, $S_{c}$ will still have positive scalar curvature since this depends only on local curvature computations. We have arrived at

Theorem 2.4. Let $M$ be a hyperkähler manifold with a locally free action of $S p(1)$ or $S O(3)$ (but not free) permuting the complex structures. Suppose further that the obstructions d $\phi$ vanish, and that the level sets of the hyperkähler potential $S_{c}=\nu^{-1}(c)$ are compact. Then $S_{c}$ is homothetic to a smooth compact 3-Sasakian manifold of positive scalar curvature. Furthermore, $S$ is a singular fibration over a compact quaternionic Kähler orbifold $M^{\prime}$ of positive scalar curvature with totally geodesic fibres of constant curvature 1 away from the orbifold singularities of $M^{\prime}$.

This theorem provides us with new examples of compact non-homogeneous Einstein manifolds of positive scalar curvature in dimension $4 n+3$. In Section 4 we give very interesting examples of compact 3-Sasakian manifolds as singular fibrations over quaternionic Kähler orbifolds. They admit a locally free (but not free) $G$ action. In particular, in dimension 7 we obtain smooth Einstein manifolds whose quotients are complex weighted projective spaces with three isolated orbifold points and with the self-dual Einstein metric constructed by Galicki and Lawson [GL]. There are plenty of examples in higher dimensions as well. Also notice that Theorem 2.1 can be generalized to the case when $M$ itself is a hyperkähler orbifold. The level sets of the hyperkähler potential function $S_{c}$ will then be Riemannian orbifolds with a 3-Sasakian structure defined away from the singular locus.

Another interesting observation comes from a theorem of Bérard-Bergery [Bes, 9.73]. 
Theorem 2.5. Let $S_{c}$ denote the level sets of $\nu$ so that the hypotheses of theorem 2.1 or theorem 2.4 are satisfied. Then there are two distinct Einstein metrics on $S_{c}$.

Proof. If the action of $G$ is free on the hyperkähler manifold $M$, then we can apply theorem 9.73 of [Bes] directly. We only need to check that the conditions of theorem 9.73 of [Bes] are satisfied for the manifolds $S_{c}$. Whereas, if the action of $G$ is only locally free, we cannot apply theorem 9.73 of [Bes] directly. However, since the level surfaces $S_{c}$ are assumed to be compact, there are a finite number of orbifold singularities on $M^{\prime}$, and if we remove these singularities together with their fibres, the result is a Riemannian submersion to which we can apply theorem 9.73 of [Bes]. The only condition in this theorem that does not follow immediately from Corollary 2.2 and 9.61 of [Bes] is condition $9.73 \mathrm{e}$. This condition is

$$
\check{\lambda}^{2}-\operatorname{dim} F(\mu+2 \nu)>0,
$$

where $|A|^{2}=\mu \cdot \operatorname{dim} F=\nu \cdot \operatorname{dim} B, B$ is the base, and $F$ is the fibre of the fibration. In our case we have $\mu=\frac{4 n \nu}{3}$, and from the proof of Corollary 2.2, we have $|A|^{2}=3 \lambda-6, \hat{s}=6$, and $s=(4 n+3) \lambda$. Now [Bes, 9.37] gives

$$
\pi^{*} \check{s}=s+|A|^{2}-\hat{s} .
$$

Combining this with $\check{r}=\check{\lambda} \check{g}$ gives

$$
\check{\lambda}=\frac{(4 n+6) \lambda-12}{4 n} \text {. }
$$

Putting this information into the left hand side of (2.9) and rearranging, one sees that condition (2.9) becomes

$$
16 n^{2}\left(\lambda^{2}-6 \lambda+12\right)+24 n(2 \lambda-3)(\lambda-2)+36(\lambda-2)^{2}>0 .
$$

This is satisfied since $0<|A|^{2}=3(\lambda-2)$. To complete the proof in the singular case we need only put back the orbifold singularities and their fibres, and notice that the metrics on $\left(S_{c^{-}}\right.$singular fibres) extend smoothly since the singular fibres have the form $G / \Gamma$ for some finite subgroup $\Gamma \subset G$. 
Remark 2.1. In the regular case Corollary 2.2 and Proposition 9.61 of [Bes] imply that the horizontal distribution $\mathcal{H}$ on $S_{c}$ defines a Yang-Mills connection. In fact, it can be checked directly that these connections are anti-self-dual in the sense of [CSal] and [GP]. Moreover, the principal bundles $Y$ constructed in Proposition 14.85 of [Bes] are precisely our manifolds $S$. However, we have seen from the local nature of [Bes, 9.73] that all of these results generalize to the case of singular fibrations over quaternionic Kähler orbifolds.

In the regular case, the level sets $S_{c}$ are also circle bundles over the twistor space $\mathcal{Z}\left(M^{\prime}\right)$. One can fix a complex structure $I$ on $M$ and view $M$ as a Kähler manifold with the associated Kähler form $\omega_{I}$. Then there is a circle subgroup of $G$ which acts on $M$ by holomorphic isometries, and, locally, is given by the vector field $X$. This gives the usual Kähler moment map $\mu_{I}$. In the case of vanishing obstructions $\mu_{I}=\nu$ coincides with the hyperkähler potential and the quotient of $\nu^{-1}(c)$ by the circle action gives the twistor space of $M^{\prime}$. We then have the following diagram of fibrations:

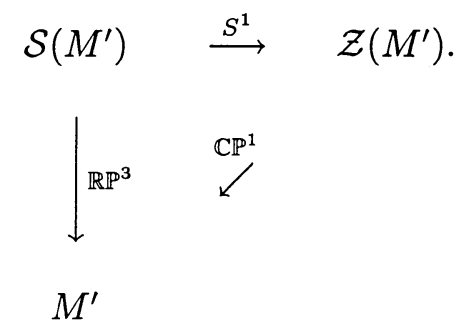

As already pointed out in Corollary $1.10 \mathcal{Z}\left(M^{\prime}\right)=\nu^{-1}(c) / S^{1}$ is an example of a Kähler-Einstein quotient and in the regular case both $M$ and $\mathcal{Z}\left(M^{\prime}\right)$ are Kähler-Einstein manifolds. The vector field $X$ defines a Sasakian structure on $S_{c}$ compatible with this fibration. In the singular case, $\mathcal{Z}\left(M^{\prime}\right)$ is a KählerEinstein orbifold and the horizontal map in (2.10) is a Seifert fibration.

This suggests the following question. Suppose we have a Kähler (but not necessarily hyperkähler) manifold $(M, g, I, \omega)$ with a holomorphic circle action by isometries. Let $X$ be the Killing vector field of this action. Consider its moment map $\mu$ and suppose $S=\mu^{-1}(c) \in M$ is a smooth manifold. Let us define $\eta(Y)=g(Y, X)$ on $M$. Consider $(\tilde{g}, \Phi, \tilde{\eta}, X)$ defined on $S$ as follows:

$$
\tilde{g}=i^{*} g, \quad \Phi=p \circ I, \quad \tilde{\eta}=i^{*} \eta,
$$


where $i$ is the inclusion map of $S$ into $M$ and $p$ is the orthogonal projection as in (2.8). Is $(\tilde{g}, \Phi, \tilde{\eta}, X)$ a Sasakian structure? Clearly not, as condition (2.1d) would then imply that the norm of the vector field $X$ is equal one on $S$. This is usually not the case and such condition turns out to be very restrictive. Now assume that this is the case. Even then condition (2.3) would not be satisfied in general. However, we will show that it is satisfied when the metrics coming from the different level sets are homothetic. This is equivalent to requiring that the normal vector $\operatorname{grad} \mu=I X$ generates a local homothety transformation on $M$ and that the complexification of $S^{1}$ acts on $M$ by homothety.

Proposition 2.6. Let $(M, g, I, \omega)$ be a Kähler manifold with an isometric and holomorphic circle action. Let $\mu$ be the associated equivariant moment map and let $S=\mu^{-1}(c)$ be a submanifold in $M$. If the norm of the vector field $X$ for the action of $S^{1}$ is constant on $S$ and $I X$ is a local homothety then $S$ is a Sasakian manifold with Sasakian structure given by (2.11).

Proof. The moment map for the $S^{1}$ action on $M$ is defined as

$$
d \mu=X\rfloor \omega, \quad \mu: M \rightarrow \mathbb{R},
$$

where $\omega$ is the Kähler form and $X$ is the holomorphic Killing vector field for the circle action. Since

$$
g(I X, Y)=\omega(X, Y)=X\rfloor \omega(Y)=d \mu(Y)=g(\operatorname{grad} \mu, Y)
$$

for each $Y \in T M$, we obtain

$$
\operatorname{grad} \mu=I X
$$

or $X=-\operatorname{grad} \mu$. The vector fields $X$ and $I X$ generate a local holomorphic action of the complexification of $S^{1}$. Let us choose $S_{c}=\mu^{-1}(c) \subset M$. This is an $S^{1}$-invariant submanifold. Since the norm of the vector field $X$ (and of $I X$ ) is constant on the level sets of the moment map, we can always normalize it so that $g(X, X)=g(I X, I X)=1$ on $S_{c}$. As $\eta$ is defined to be the contraction of the metric with $X, \eta(Y)=g(Y, X)$, the condition $\tilde{\eta}(X)=1$ is satisfied trivially. Also

$$
\Phi(X)=p \circ I(X)=I X-\eta(X) \operatorname{grad} \mu=0
$$


and

$$
\eta(\Phi Y)=g(\Phi Y, X)=g(I Y-\eta(Y) \operatorname{grad} \mu, X)=g(I Y, X)=-g(Y, I X)=0
$$

for each $Y \in T S_{c}$. Similarly

$$
\begin{aligned}
\Phi \circ \Phi(Y) & =I(\Phi Y)-\eta(\Phi Y) \operatorname{grad} \mu \\
& =I(I Y-\eta(Y) \operatorname{grad} \mu)=-Y+\eta(Y) X, \quad Y \in T S_{c}
\end{aligned}
$$

Hence, $\left(i^{*} g, X, \Phi, i^{*} \eta\right)$ is an almost contact structure on $S_{c}$. This structure is compatible with the metric as

$$
g(\Phi Y, \Phi Z)=g(I Y-\eta(Y) \operatorname{grad} \mu, I Z-\eta(Z) \operatorname{grad} \mu=g(Y, Z)-\eta(Y) \eta(Z) .
$$

Moreover, it is a normal almost contact metric structure and the proof of normality is identical to the one in Thereom 2.1 .

Notice that we have not used the assumption that grad $\mu$ generates a local homothety transformation. We need this to show that the almost contact normal metric structure $\left(i^{*} g, X, \Phi, i^{*} \eta\right)$ is contact; i.e., $S_{c}$ is a Sasakian manifold. If $I X$ is a local homothety then

$$
\mathcal{L}_{I X} \omega=\lambda \omega
$$

Without loss of generality we can assume that $\lambda=-1$, as one can always rescale both the vector field $X$ and the metric $g$ on $M$ in such a way that the new $X$ has still norm one in the new metric but $\lambda$ is any constant. Notice that

$$
\eta(Y)=g(Y, X)=g(I Y, I X)=\omega(Y, I X)=-(I X\rfloor \omega)(Y), \quad Y \in T M
$$
or $\eta=-I X\rfloor \omega$. Hence,

$$
d \eta=-d(I X\rfloor \omega)=-\mathcal{L}_{I X} \omega=\omega
$$

and $d \tilde{\eta}=d i^{*} \eta=i^{*} \omega$. This is enough to show that (2.3) holds and that the almost contact structure is contact.

The proposition above shows that there is a natural Sasakian geometry associated to any Kähler-Einstein quotient and many new Sasakian manifolds can be obtained this way [PePo1]. 


\section{The Twistor Space}

We now reinterpret Theorem 1.4 in terms of twistor geometry. Our main result in this regard is theorem 3.2 below. We begin by setting notation. Let $\mathcal{O}(k)$ denote the $k$-fold tensor product of the hyperplane bundle on the complex projective line $\mathbb{P}^{1}$ or on any rational curve $\mathbb{P}^{1} \rightarrow \mathcal{Z}$ embedded in a complex manifold $\mathcal{Z}$. Note that $\mathcal{O}=\mathcal{O}(0)$ is the trivial line bundle and $\mathcal{O}(-1)$ is the dual tautological bundle. If $E$ is a holomorphic vector bundle on $\mathcal{Z}$ we can "twist" $E$ with $\mathcal{O}(k)$ to get new vector bundles $E \otimes \mathcal{O}(k)$ which are denoted by $E(k)$.

Recall that on any quaternionic manifold $M$ there is the locally defined vector bundle $H$ corresponding to the standard complex representation of $S p(1)$ discussed in Section 1. Although $H$ is, in general, only defined locally, the symmetric product bundle $S^{2} H$ and the projective bundle $\mathbb{P}(H)$ are both globally defined on $M$. Furthermore, the representation spaces $S^{2} H$ can be identified with the adjoint representation $\mathfrak{s p}(1)$ of $S p(1)$. This is a 3-dimensional real vector bundle on $M$ whose unit sphere bundle $\mathcal{Z}(M)$, which is called the twistor space of $M$, is of basic interest. Indeed, Salamon [Sal1, Sal2] has shown that, if $M$ is a quaternionic manifold, then $\mathcal{Z}(M)$ is a complex manifold fibered by rational curves. There are converses to Salamon's result, given in $[\mathrm{PePo} 2, \mathrm{~L}]$ for the general quaternionic case, and in [HKLR] for the hyperkähler case.

More precisely, Salamon's theorem shows that the twistor space $\mathcal{Z}=\mathcal{Z}(M)$ of any quaternionic manifold $M$ is a complex manifold of complex dimension $2 n+1$ satisfying the following properties:

(i) There is an antiholomorphic fixed point free involution $\tau$ on $\mathcal{Z}$, called a real structure.

(ii) $\mathcal{Z}$ is fibered by rational curves that are $\tau$-invariant, called real twistor lines.

(iii) The normal bundle $N$ of any real twistor line is isomorphic to $\mathbb{C}^{2 n} \otimes$ $\mathcal{O}(1)$.

Conversely, if $\mathcal{Z}$ is any complex manifold of complex dimension $2 n+1$ that satisfies conditions (i)-(iii), then it is the twistor space of a quaternionic 
manifold $M$ of real dimension $4 n$ where the quaternionic manifold $M$ is the space of all real twistor lines in $\mathcal{Z}$, [Sal1, PePo2, L, HKLR]. There are two distinct cases to consider:

Case 3.1. Non-zero scalar curvature [PePo2, L]: To obtain a (pseudo)-quaternionic Kähler structure with nonzero scalar curvature on $M$, we must have a complex contact structure on $\mathcal{Z}$ compatible with the real structure, and such that the contact distribution $D$ is transverse to the real twistor lines. This is given by a 1 -form $\theta$ with values in the contact line bundle $L$ on $\mathcal{Z}$ such that $\theta \wedge(d \theta)^{n}$ is a non-degenerate holomorphic $2 n+1$ form. This then determines a quaternionic Kähler metric on $M$. Furthermore, if this metric has positive scalar curvature, then $\mathcal{Z}(M)$ has a Kähler-Einstein metric [Sal1].

Case 3.2. Zero scalar curvature [HKLR]: In this case there is a holomorphic fibration

$$
p: \mathcal{Z} \rightarrow \mathbb{P}^{1}
$$

and, instead of a contact structure on $\mathcal{Z}$, there is a holomorphic section $\kappa$ of $\Lambda^{2} T_{F}^{*}(2)$ defining a complex symplectic structure on each fibre $F$ of $p$. Furthermore, each fibre is diffeomorphic to a manifold $M$ whose points are the fixed point set of the real structure $\tau$. Here $\mathcal{Z}$ is diffeomorphically, but not holomorphically, a product $M \times S^{2}$. Again the hyperkähler metric on $M$ is determined from $\kappa$.

To tie this into our discussions in Section 1 we consider the case that $M$ has a given hyperkähler metric $g$ with complex structures $I^{a}$. The complex structure on $\mathcal{Z}$ is given by $\mathfrak{I}=\left(x \cdot I, I_{0}\right)$ where $x \in S^{2}$ is written in standard Cartesian coordinates in $\mathbb{R}^{3}, I \in \mathcal{I}$, and $I_{0}$ is the standard complex structure on the Riemann sphere $\mathbb{P}^{1}$. Now suppose, as in section Section 1 , that $M$ admits a locally free action of $G$ that acts nontrivially on the bundle of complex structures $\mathcal{I}$ (or equivalently on $\mathcal{G}$ ), and consider the standard action of $S O(3)$ on $S^{2}$. As before let $X^{a}$ denote the infinitesimal generators of the $G$ action on $M$ corresponding to the standard basis in $\mathfrak{s p}(1)$. Let $\xi^{a}$ denote the infinitesimal generators for the standard $S O(3)$ action on $S^{2}$, then

$$
Y^{a}=X^{a}+\xi^{a}
$$


are easily seen to be infinitesimal automorphisms of the complex structure $\mathfrak{I}$ on $\mathcal{Z}$. Hence, there is an holomorphic action of $G$ on the twistor space $\mathcal{Z}(M)$. Furthermore, since under $p$ this action projects to a transitive action of $S O(3)$ on the 2-sphere of complex structures on $M$, we have

Proposition 3.1. Let $(M, g)$ be a hyperkähler manifold on which there is an action of $S p(1)$ or $S O(3)$ by isometries which act non-trivially on the bundle $\mathcal{I}$. Then all the complex structures $I \in \mathcal{I}$ are holomorphically equivalent.

For each point $x \in \mathbb{P}^{1}, M_{x} \simeq p^{-1}(x)$ is a divisor on $\mathcal{Z}$, and Proposition 3.1 says that all these divisors are biholomorphically equivalent. Furthermore, each divisor $M_{x}$ has a Kähler structure given by $\omega_{x}=x \cdot \omega=\sum x^{a} \omega^{a}$, and the $S O(3)$ action taking $x$ to $x^{\prime}$ carries the Kähler structure on $M_{x}$ to the Kähler structure on $M_{x^{\prime}}$. The stabilizer in $S O(3)$ of a divisor $M_{x}$ with its Kähler structure $\omega_{x}$ is a circle group $U(1)_{x}$. This gives rise to a Kähler moment map

$$
\mu_{x}: M_{x} \longrightarrow \mathbb{R},
$$

which, using Theorem 1.4, is easily seen to be given by

$$
\mu_{x}=\nu+x \cdot \phi \cdot x .
$$

The Marsden-Weinstein quotient $\mathcal{Z}_{x}^{\prime}=\mu_{x}^{-1}(c) / U(1)_{x}$ is a Kähler manifold with Kähler form induced from $\omega_{x}$. In the case that the obstructions $d \phi$ vanish, we can take $\mu_{x}=\nu$, and thus the $U(1)$ moment map $\mu_{x}$ on $M$ is independent of the complex structure. Since the stability subgroups $U(1)_{x}$ are all isomorphic, the quotient $\mathcal{Z}^{\prime}=\nu^{-1}(c) / U(1)$ is independent of $x$. Furthermore, since the level sets of $\mu_{x}=\nu$ are $G$ invariant, we obtain a fibration of $\mathcal{Z}^{\prime}=\nu^{-1}(c) / U(1)$ over the quaternionic Kähler manifold $\nu^{-1}(c) / G$ with fibres $\mathbb{P}^{1}$. This fibration is contained in diagram (0.4). One can then check that $\mathcal{Z}^{\prime}$ is the twistor space of $\nu^{-1}(c) / G$. However, if the obstructions $d \phi$ do not vanish, then $G$ does not act on the level sets of the Kähler moment map $\mu_{x}$; hence, no such fibering of $\mathcal{Z}_{x}^{\prime}$ by rational curves exists, and $\mathcal{Z}_{x}^{\prime}$ is not the twistor space of a quaternionic Kähler manifold.

Let $T^{(1,0)}$ denote the sheaf of germs of holomorphic vector fields of type $(1,0)$ on $\mathcal{Z}$. Since the vector $Y^{a}$ defined in (3.2) are infinitesimal automorphisms 
of the complex structure $\mathfrak{I}$ on $\mathcal{Z}$, the vector fields $Z^{a}=\left(Y^{a}-i \mathfrak{I} Y^{a}\right) / 2$ are holomorphic. Consider the sheaf $\mathcal{O}(2)$ on $\mathbb{P}^{1}$, it has 3 linearly independent sections which we can take to be

$$
Q^{1}=2 \zeta^{1} \zeta^{2}, \quad Q^{2}=\left(\zeta^{2}\right)^{2}-\left(\zeta^{1}\right)^{2}, \quad Q^{3}=i\left(\left(\zeta^{1}\right)^{2}+\left(\zeta^{2}\right)^{2}\right)
$$

Then the twisted complex symplectic form $\kappa$ along the fibres of $p$ is given by

$$
\kappa=Q \cdot \omega=\sum Q^{a} \omega^{a},
$$

and we can define a twisted holomorphic vector field on $\mathcal{Z}$; that is, a section of $T^{(1,0)}(2)$, by

$$
\Psi=Q \cdot Z=\sum Q^{a} Z^{a} .
$$

Thus, $\tilde{\phi}=\Psi\rfloor \kappa$ defines a holomorphic section of $\Lambda^{(1,0)}(4)$. But, since $\kappa$ is a holomorphic $(2,0)$ form along the fibres of $p$, and since the sections $Q$ in (3.5) lie on the quadric cone $Q^{2}=0$, we have

$$
\tilde{\phi}=Q \cdot Z\rfloor \kappa=Q \cdot X\rfloor \kappa=Q \cdot X\rfloor \omega \cdot Q=Q \cdot d \phi \cdot Q .
$$

This implies the main result of this section:

Theorem 3.2. The Kähler manifold $\mathcal{Z}_{x}^{\prime}=\mu_{x}^{-1}(c) / U(1)_{x}$ is the twistor space of a quaternionic Kähler manifold with positive scalar curvature if and only if

$$
\tilde{\phi} \in H^{0}\left(\mathcal{Z}, \Lambda^{(1,0)}(4)\right)
$$

vanishes.

\section{EXAMPLES}

In this section we give explicit examples of quaternionic Kähler reductions to show the scope of the theory analyzed in Section 1.

EXAMPLE 4.1. QUATERNIONIC PROJECTIVE SPACE.

The model example of the $S p(1)$ quotient is that of the $n$-dimensional quaternionic vector space $\mathbb{H}^{n}$. Let $\vec{u} \in \mathbb{H}^{n}$ and let $\vec{u}^{*}$ be its quaternionic conjugate. We define the flat metric $g=\operatorname{Re}\left\{d \vec{u}^{*} \otimes d \vec{u}\right\}$ and the hyperkähler structure $\omega=\frac{1}{2} \operatorname{Im}\left\{d \vec{u}^{*} \otimes d \vec{u}\right\}=\sum_{a=1}^{3} \omega^{a} e_{a}$, where $\left\{e_{a}\right\}_{a=1,2,3}=\{i, j, k\}$ is 
the standard basis for the unit quaternions. If we introduce real coordinates $\vec{u}=\vec{\tau}+\vec{x}^{a} e_{a}$ then

$$
\omega^{a}=\sum_{\alpha=1}^{n}\left(d \tau_{\alpha} \wedge d x_{\alpha}^{a}-\frac{1}{2} \epsilon^{a b c} d x_{\alpha}^{b} \wedge d x_{\alpha}^{c}\right) .
$$

First, let us consider the action of $S p(1)$ on $\mathbb{H}^{n}$ by the scalar multiplication from the right,

$$
\mathbb{H}^{n} \ni \vec{u} \stackrel{\sigma}{\rightarrow} \vec{u} \sigma \quad \text { for } \quad \sigma \in S p(1)
$$

This is an isometric action rotating the quaternionic structure as in (1.3); that is, $\omega \stackrel{\sigma}{\mapsto} \bar{\sigma} \omega \sigma$. The action is free on $\mathbb{H}^{n} \backslash\{0\}$. Locally, it is generated by $X=X^{a} e_{a}=\operatorname{Im}\left(\vec{u}^{* t} \frac{\partial}{\partial \vec{u}}\right)$ where $\frac{\partial}{\partial \vec{u}}=\frac{\partial}{\partial \vec{\tau}}+e_{a} \frac{\partial}{\partial \vec{x}^{a}}$. In real coordinates we have

$$
X^{a}=\sum_{\alpha=1}^{n}\left(\tau_{\alpha} \frac{\partial}{\partial x_{\alpha}^{a}}-x_{\alpha}^{a} \frac{\partial}{\partial \tau_{\alpha}}-\frac{1}{2} \epsilon^{a b c} x_{\alpha}^{b} \frac{\partial}{\partial x_{\alpha}^{c}}\right) .
$$

With this choice of normalization $\left[X^{a}, X^{b}\right]=2 \epsilon^{a b c} X^{c}$. A direct calculation shows that $\nu=\frac{1}{2} \vec{u}^{* t} \vec{u}, \phi \equiv 0$, and $\eta=\operatorname{Im}\left(\vec{u}^{* t} d \vec{u}\right)$. Clearly, $\eta^{b}\left(X^{a}\right)=2 \nu \delta^{a b}$. In this case $M^{\prime}=\nu^{-1}(c) / S p(1) \simeq \mathbb{H} \mathbb{P}^{n-1}$ and the quotient metric is the standard one. The $S p(1)$ action extends to a homothetic $\mathbb{H}^{*}$ action and $M^{\prime}=$ $\nu^{-1}(c) / S p(1) \simeq \mathbb{H}^{n} \backslash\{0\} / \mathbb{H}^{*}$ where $\mathbb{H}^{n} \backslash\{0\}$ is the $\mathbb{Z} / 2$ lift of the associated quaternionic bundle over $\mathbb{H}^{n-1}$ in the sense of Swann [Sw1, Sw2].

One can take any $U(1) \subset S p(1)$ and projectivize $\mathbb{H}^{n} \backslash\{0\} \simeq \mathbb{C}^{2 n} \backslash\{0\}$ with respect to this particular $U(1)$. Such projectivization gives the twistor space $\mathcal{Z}$ of $\mathbb{H}^{n-1}$ which, in our case, is $\mathbb{C P}^{2 n-1}$. $\mathcal{Z}$ carries its Fubini-Study metric. It is Kähler-Einstein and $\mathbb{C P}^{2 n-1}$ can be viewed as the Kähler-Einstein quotient of the hyperkähler manifold $\mathbb{H}^{n} \backslash\{0\}$. We have the following diagram:

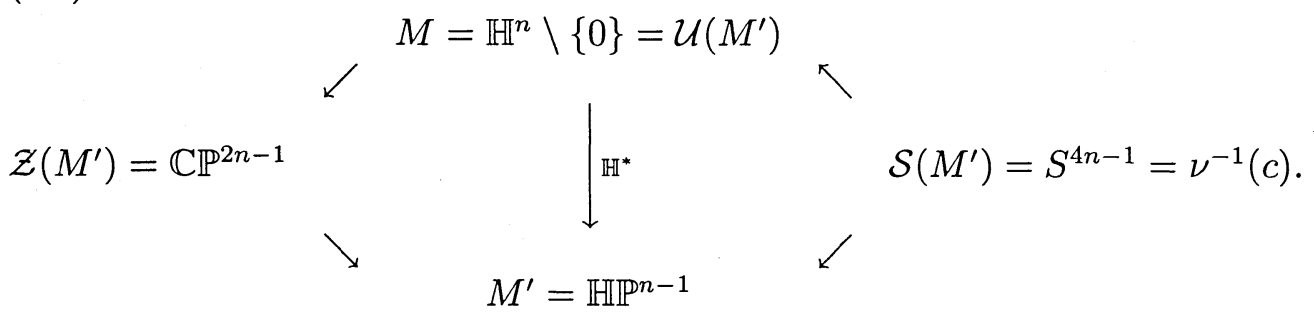


The level sets of the momentum map $\nu$ are $(4 n-1)$-dimensional spheres with the standard symmetric Einstein metric. They are model examples of a 3Sasakian manifold, with a 3-Sasakian structure defined by the vector fields (4.3) [Sas1]. The $(4 n-1)$-dimensional sphere fibers over $\mathbb{H}^{\mathbb{P}^{n-1}}$ and the projection map is the 3-Sasakian submersion. In this case it is just the quaternionic Hopf fibration. But $S^{4 n-1}$ fibers also over the complex projective space $\mathbb{C P}^{2 n-1}$ as a circle bundle. This is the standard Hopf fibration. The complex projective space $\mathbb{C P}^{2 n-1}$ is the twistor space of $\mathbb{H} \mathbb{P}^{n-1}$ with fiber $\mathbb{C P}^{1}$. We have the following diagram of fibrations:

$$
\begin{array}{lll}
S^{4 n-1} & \stackrel{S^{1}}{\longrightarrow} & \mathbb{C P}^{2 n-1} . \\
S^{3} & \mathbb{C P}^{1} \\
\mathbb{H}^{n-1} &
\end{array}
$$

It is well-known that there exist a second $S p(n+1)$-invariant homogeneous Einstein metric on $S^{4 n-1}$ which is not isometric to the standard round sphere metric [Bes]. For $n \neq 4$ there are no other homogeneous Einstein metrics on $S^{4 n-1}$.

Next, we will present two examples of hyperkähler manifolds with $S O(3)$ action by isometries rotating the hyperkähler structure and with non-vanishing obstruction $\phi$.

Example 4.2. Flat Quaternionic VeCtor SPACE REVisited.

Let $M=\mathbb{H}^{n}$ with the same flat metric and the hyperkähler structure as before but now consider the adjoint action of $S p(1)$ on $M$; that is,

$$
\mathbb{H}^{n} \ni \vec{u} \stackrel{\sigma}{\rightarrow} \vec{\sigma} \vec{u} \sigma \quad \text { for } \quad \sigma \in S p(1) .
$$

Here $\mathbb{Z} / 2$ acts trivially so we get an $S O(3)$ action on $M$. It is an isometry rotating the quaternionic structure just as in Example 1. The action is not locally free but let us restrict our attention to the subset of $M$ where the action of $S O(3)$ is free defined as a union of the orbits with trivial isotropy group. This is an open dense set in $\mathbb{H}^{n}$ (for $n>1$ ) and it is a hyperkähler 
manifold. Choose the basis for the $S O(3)$ such that $X^{a}=\frac{1}{2}\left(-e_{a} \vec{u}+\vec{u} e_{a}\right)$. A simple calculation shows that

$$
\begin{aligned}
\nu & =\frac{1}{3}\|\operatorname{Im}(\vec{u})\|^{2}=\frac{1}{3} \sum_{\alpha=1}^{n}\left(x_{\alpha}^{a}\right)^{2}, \\
\phi^{a b} & =\frac{1}{2} \sum_{\alpha=1}^{n}\left(x_{\alpha}^{a} x_{\alpha}^{b}+\nu \delta^{a b}\right), \\
\eta^{a} & =-\sum_{\alpha=1}^{n}\left(x_{\alpha}^{a} d \tau_{\alpha}+\frac{1}{2} \epsilon^{a b c} x^{b} d x^{c}\right) .
\end{aligned}
$$

As $\phi$ is not constant on $\nu^{-1}(c)$ the quotient $M^{\prime}=\nu^{-1}(c) / S O(3)$ is not a quaternionic Kähler manifold.

Notice that we can mix the two actions on the different coordinates of $\mathbb{H}^{n}$. Any such $S p(1)$ action would rotate the qual, phionic structure as in (1.3) but $\phi$ would not be constant on $\nu^{-1}(c)$.

EXAmple 4.3. The generalized TAUB-NUT MEtric.

Let $M$ be the $4 n$-dimensional analogue of the Taub-NUT hyperkähler metric. Again, we will introduce $M$ as a hyperkähler quotient of the flat space $\mathbb{H}^{n+1}$. There is a free $\mathbb{R}$ action on $\mathbb{H}^{n+1}$ by hyperkähler isometries

$$
\mathbb{H}^{n+1} \ni(\vec{u}, w) \stackrel{t}{\mapsto}\left(e^{i t} \vec{u}, w+t\right) \quad \text { for } \quad t \in \mathbb{R} .
$$

The moment map $\mu: \mathbb{H}^{n+1} \rightarrow \mathbb{R}$ is given by

$$
\mu(\vec{u}, w)=\vec{u}^{* t} i \vec{u}+2 \operatorname{Im}(w) .
$$

The zero-level set $\mu^{-1}(0) \subset \mathbb{H}^{n+1}$ is a submanifold and the quotient $M=$ $\mu^{-1}(0) / \mathbb{R}$ carries a natural hyperkähler structure. The isometry group of $M$ is $U(n) \times S p(1)$, where $U(n)$ acts preserving the hyperkähler structure and $S p(1)$ by rotating it. The $S p(1)$ action can be described explicitly as

$$
M \ni[\vec{u}, w] \stackrel{\sigma}{\mapsto}[\vec{u} \sigma, \bar{\sigma} w \sigma] \quad \text { for } \quad \sigma \in S p(1)
$$

where $[\vec{u}, w]$ is the "homogeneous" coordinate on $M$. Notice that $\mathbb{Z} / 2$ subgroup acts trivially. Example 4.2 shows that this action has a nontrivial obstruction $\phi$. 
Many examples of hyperkähler manifolds with $S O(3)$ action rotating the hyperkähler structure and with vanishing obstruction $\phi$ can be obtained from flat space with the right action described in Example 4.1. This is an observation due to Swann [Sw1] which makes use of the hyperkähler reduction procedure of [HKLR].

Let $M$ be a hyperkähler manifold satisfying the hypothesis of the Theorem 1.4. Let $H$ be a compact Lie group of hyperkähler isometries of $M$ and such that $H$ commutes with the $S p(1)$ (or $S O(3)$ ) action. Suppose $H$ defines an equivariant momentum mapping $\mu: M \rightarrow \mathfrak{h}^{*} \otimes \mathbb{R}^{3}$ such that $0 \in \mathfrak{h}^{*} \otimes \mathbb{R}^{3}$ is its regular value. If $H$ acts freely on $\mu^{-1}(0) \subset M$ then the quotient $\hat{M}=$ $\mu^{-1}(0) / H$ is again a hyperkähler manifold. It is the hyperkähler reduction of $M$ by $H$. Suppose that the moment map $\mu$ is chosen in such a way that $\mu^{-1}(0)$ is an $S O(3)$ (or $S p(1)$ ) invariant submanifold in $M$. This can always be done, for example, when $H^{1}(M, \mathbb{R})=0$ and $H$ is Abelian. In this case $\mu$ can be written as

$$
\langle\mu(m), \zeta\rangle=\eta\left(\zeta^{*}\right), \quad \zeta \in \mathfrak{h}, m \in M,
$$

where $\eta$ is the $\mathfrak{s p}(1)$-valued one-form defined in (1.2) and $\zeta^{*}$ is the vector field for the $H$-action associated to $\zeta$. Also, $H$ projects to a quaternionic isometry of the quaternionic Kähler base $M^{\prime}$. One can see that the reduced space $\hat{M}$ satisfies the hypothesis of the Theorem 1.4 and we have the commutative diagram (0.5). The commutativity follows from a theorem of Swann [Sw1, Sw2]. He observed that the quaternionic Kähler quotient of Galicki and Lawson [GL] is simply the hyperkähler quotient of Hitchin et. al. [HKLR] in the associated quaternionic bundle $\mathcal{U}\left(M^{\prime}\right)$ with respect to the zero level set. But our $M$ is locally homothetic to the associated quaternionic bundle $\mathcal{U}\left(M^{\prime}\right)$.

Let us further remark that the diagram (0.5) can be used to define a reduction of fibered Riemannian 3-Sasakian spaces by certain group of isometries. This reduction is an analogue of the hyperkähler reduction.

\section{EXAMPLE 4.4. WOLF SPACES AND NILPOTENT ADJOINT ORBITS.}

Let $M$ be the highest weight nilpotent orbit $\mathcal{O}_{n+1}$ of $S L(n+1, \mathbb{C})$ acting by the adjoint action on its Lie algebra of traceless endomorphisms of $\mathbb{C}^{n}$. There 
is a very simple model of $\mathcal{O}_{n+1}$ as a hyperkähler quotient of the flat space $\mathbb{H}^{n+1} \backslash\{0\}$. Consider the hyperkähler $U(1)$ action on $\mathbb{H}^{n+1} \backslash\{0\}$ defined as

$$
\mathbb{H}^{n+1} \backslash\{0\} \ni \vec{u} \stackrel{t}{\mapsto} e^{i t} \vec{u} \quad \text { for } \quad t \in[0,2 \pi) .
$$

Such an action is free and gives the hyperkähler moment map $\mu: M \rightarrow \mathbb{R}^{3} \simeq$ $\mathfrak{s p}(1)^{*}$ defined by

$$
\mu(\vec{u})=\vec{u}^{* t} i \vec{u}
$$

Now, $\mu^{-1}(0) \subset \mathbb{H}^{n+1} \backslash\{0\}$ is a $U(1)$-invariant open submanifold and the quotient $\mathcal{O}_{n+1}=\mu^{-1}(0) / U(1)$ can be easily seen to be diffeomorphic to the highest weight nilpotent adjoint orbit of $S L(n+1, \mathbb{C})$. In fact, the quotient metric is hyperkähler, although it is incomplete. $\mathcal{O}_{n+1}$ admits an $S O(3)$ action rotating the hyperkähler structure. One can write this action as

$$
\mathcal{O}_{n+1} \ni[\vec{u}] \stackrel{\sigma}{\mapsto}[\vec{u} \sigma] \quad \text { for } \quad \sigma \in S p(1)
$$

where $[\vec{u}]$ is a "homogeneous coordinate" on $\mathcal{O}_{n+1}$. Now $S O(3)$ extends to a homothetic action by $\mathbb{H}^{*} /(\mathbb{Z} / 2)$ and the obstruction $\phi$ vanishes. The quotient is a smooth compact quaternionic Kähler manifold of positive scalar curvature; more precisely, the complex symmetric Wolf space

$$
\begin{aligned}
M^{\prime}=\nu^{-1}(c) / S O(3) & \simeq \mathcal{O}_{n+1} /\left(\mathbb{H}^{*} / \mathbb{Z} / 2\right) \\
& \simeq X(n-1)=\frac{U(n+1)}{U(n-1) \times U(2)}=\mathbb{G}_{2, n+1}(\mathbb{C}) .
\end{aligned}
$$

Furthermore, one can fix any $U(1) \subset S p(1)$ and take the Kähler quotient by that $U(1)$. This gives the twistor space

$$
\mathcal{Z}\left(M^{\prime}\right)=F_{1,2, n+1} \simeq \frac{U(n+1)}{U(n-1) \times U(1) \times U(1)}
$$

of $X(n-1)$ with its homogeneous Kähler-Einstein metric. Again, the compact complex flag $F_{1,2, n+1}$ can be viewed as the Kähler-Einstein quotient of the incomplete hyperkähler metric on $\mathcal{O}_{n+1}$. The 3-Sasakian manifold fibering over both $\left.\mathbb{G}_{2, n+1}(\mathbb{C})\right)$ and $F_{1,2, n+1}$ is the homogeneous Einstein space

$$
\mathcal{S}\left(M^{\prime}\right) \simeq \frac{U(n+1)}{U(n-1) \times U(1)}
$$


This particular example can be understood in terms of the $U(1)$ reduction of the diagram (0.5) for the flat space:

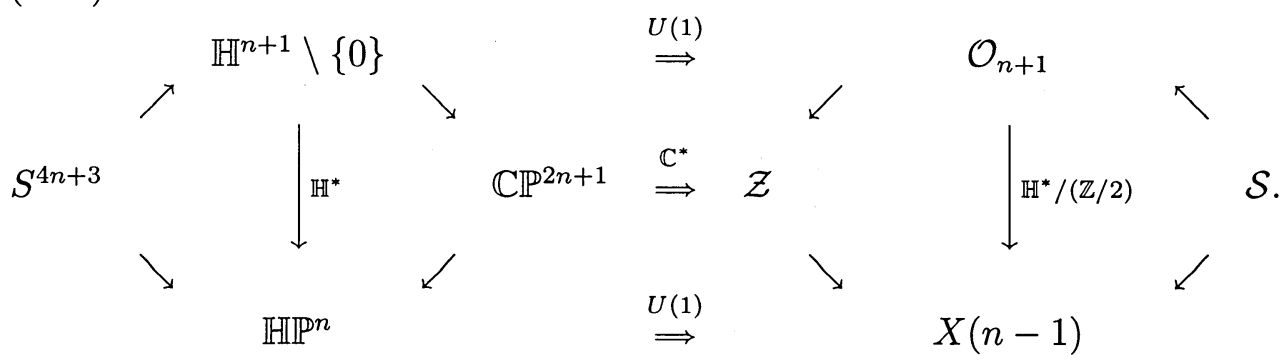

The 3-Sasakian manifold $S=\mathcal{S}(X(n-1))$ is a compact homogeneous Einstein manifold of positive scalar curvature. It is a hypersurface in the nilpotent orbit $\mathcal{O}_{n+1}$. One can also think of $\mathcal{S}$ as a 3-Sasakian reduction of the $(4 n+3)$ dimensional sphere by a circle action.

Using the $S p(1)$ reduction of $\mathbb{H}^{n+4} \backslash\{0\}$ one can construct similar diagram of fibrations over the Grassmannian $G_{n, 4}(\mathbb{R})$.

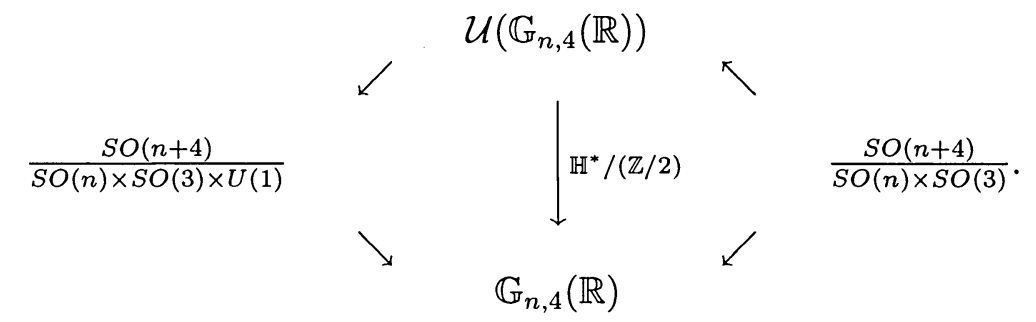

In general, any compact homogeneous symmetric Wolf space $M$ can be written as $M=K / N(S p(1))=K / L \cdot S p(1)$ [Wo], where $K$ is a compact semisimple Lie group and $N(S p(1))$ is the normalizer of $S p(1)$ in $K$. We get the following fibrations

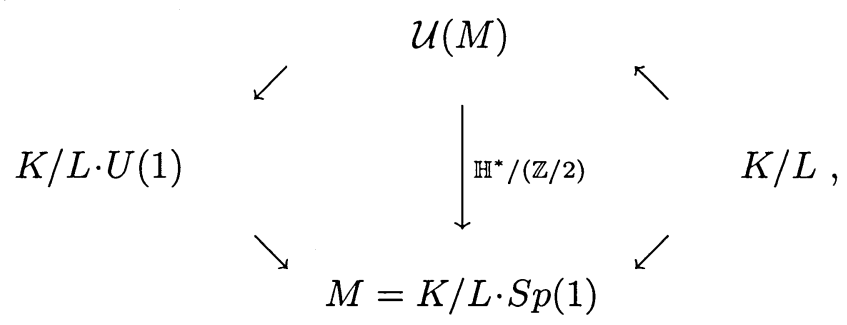

where $\mathcal{U}(M)$ is highest weight nilpotent adjoint orbit of $K^{\mathbb{C}}$ [Sw1, Sw2]. 
The fibrations

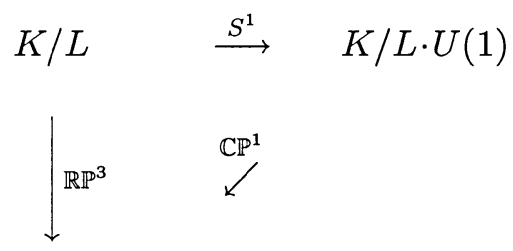

$$
K / L \cdot S p(1)
$$

are well-known [Bes]. In fact, $K / L$ is known to admit two different Einstein metrics, one of which is given by our construction. The diagram (4.21) is an example of the fibrations described in (0.4). When $K$ is an exceptional Lie group, $G_{2}, F_{4}, E_{6}, E_{7}$, or $E_{8}$, and under some additional restrictions on the geometry of the quotient, diagram (4.20) cannot be obtained as a reduction of the flat space example 4.4 [Sw1].

The example above is of particular interest as it relates to the geometry of nilpotent variety. It was recently shown by Kronheimer [Kr3] that for any compact, connected, semisimple Lie group $K$, a nilpotent adjoint orbit of its complexification $K^{\mathbb{C}}$ has a natural hyperkähler structure. Furthermore, such orbits admit a homothetic $\mathbb{H}^{*}$ action and the quotient of any orbit by that action $\mathcal{O} / \mathbb{H}^{*}$ is a quaternionic Kähler manifold [Sw1]. The complex projectivization of the orbit $\mathcal{O}$ is the twistor space of $\mathcal{O} / \mathbb{H}^{*}$. $\mathcal{O}$ has a hyperkähler potential function $\nu$. It follows from our theory that

Proposition 4.1. The level sets of the hyperkähler potential function $\nu$ in $\mathcal{O}$ are 3-Sasakian manifolds. In particular the metric induced on $\nu^{-1}(c)$ from the hyperkähler metric on $\mathcal{O}$ is Einstein and has positive scalar curvature.

Besides the (4n-1)-dimensional sphere, the only known complete 3-Sasakian manifolds obtained this way can be realized as hypersurfaces in the highest weight nilpotent orbit of $K^{\mathbb{C}}$, where $K$ is the group of a isometries of compact symmetric Wolf space. They are $\mathbb{R P}^{3}$ bundles over Wolf spaces and are homogeneous. We do not know if there are any compact examples coming from other nilpotent orbits. 
EXAmple 4.5. CompleX Weighted PROJeCtive SPACES.

Let $M=\mathbb{H}^{3} \backslash\{0\}$. Consider the following circle action on $M$ :

$$
\mathbb{H}^{3} \backslash\{0\} \ni\left(u_{1}, u_{2}, u_{3}\right) \stackrel{\tau}{\mapsto}\left(\tau^{p} u_{1}, \tau^{q} u_{2}, \tau^{s} u_{3}\right),
$$

where $\tau \in S^{1}$ and $(p, q, s)$ are pairwise coprime positive integers. The action above is hyperkähler and descends to a quaternionic Kähler action on the projective space $\mathbb{H}^{2}$. Let us describe the hyperkähler quotient of $\mathbb{H}^{3} \backslash\{0\}$ by this action. The momentum map $\mu$ is

$$
\mu\left(u_{1}, u_{2}, u_{3}\right)=p \bar{u}_{1} i u_{1}+q \bar{u}_{2} i u_{2}+s \bar{u}_{3} i u_{3} \in \mathbb{R} \otimes \mathfrak{s p}(1) .
$$

The circle action is free on $\mu^{-1}(0)$. The quotient $\hat{M}_{p, q, s}=\mu^{-1}(0) / S^{1}$ is an 8-dimensional hyperkähler manifold. It is the associated bundle of the quaternionic reduction of $\mathbb{H}^{2} \mathbb{P}^{2}$ by the circle action above. The quaternionic Kähler reduction of $\mathbb{H}^{2}$ is obtained as follows. The hyperkähler momentum map $\mu$ of (4.23) descends to the quaternionic Kähler map

$$
\tilde{\mu}\left(\left[u_{1}, u_{2}, u_{3}\right]\right)=p \bar{u}_{1} i u_{1}+q \bar{u}_{2} i u_{2}+s \bar{u}_{3} i u_{3} \in \mathbb{R} \otimes \mathcal{G} .
$$

It follows from the work of Galicki and Lawson [GL] that the quotient

$$
\tilde{\mu}^{-1}(0) / S^{1}= \begin{cases}\mathbb{C P}_{p+q, p+s, s+q}^{2}, & \text { if }(p+q+s) \text { is even; } \\ \mathbb{C P}_{\frac{p+q}{2}, \frac{p+s}{2}, \frac{s+q}{2}}^{2}, & \text { if }(p+q+s) \text { is odd. }\end{cases}
$$

This is a self-dual and Einstein 4-orbifold with positive scalar curvature and with the singular locus consisting of three isolated orbifold points except when $p=q=1$. The hyperkähler quotient $\hat{M}_{p, q, s}=\mathcal{U}\left(\mathbb{C P}_{p+q, p+s, s+q}^{2}\right)(p+q+s$ even $)$ is just the associated quaternionic bundle (at least away from the orbifold singularities).

Let us consider the level sets of the hyperkähler potential function $\hat{\nu}$ on the manifold $\hat{M}_{p, q, s}=\mathcal{U}\left(\mathbb{C P}_{p+q, p+s, s+q}^{2}\right)$. We denote by $\llbracket u_{1}, u_{2}, u_{3} \rrbracket$ the coordinates on $\hat{M}_{p, q, s}$. Then the hyperkähler potential function $\hat{\nu}$ is given by

$$
\hat{\nu}\left(\llbracket u_{1}, u_{2}, u_{3} \rrbracket\right)=\left(\bar{u}_{1} u_{1}+\bar{u}_{2} u_{2}+\bar{u}_{3} u_{3}\right) .
$$

A level set of $\hat{\nu}$

$$
S_{p, q, s}=\mathcal{S}\left(\mathbb{C P}_{p+q, p+s, s+q}^{2}\right)=\left\{\llbracket u_{1}, u_{2}, u_{3} \rrbracket \in \hat{M}_{p, q, s} \mid \hat{\nu}\left(\llbracket u_{1}, u_{2}, u_{3} \rrbracket\right)=c\right\}
$$


is a smooth compact 7-manifold. It is 3-Sasakian and therefore Einstein. But it is not a fibered Riemannian space. It admits an $S p(1)$ action which is locally free but not free and the quotient

$$
S_{p, q, s} / S p(1)=\mathbb{C P}_{p+q, p+s, s+q}^{2}
$$

is a Riemannian orbifold. The projection map

$$
S_{p, q, s} \rightarrow \mathbb{C P}_{p+q, p+s, s+q}^{2}
$$

is a singular orbifold fibration. We have the following diagram

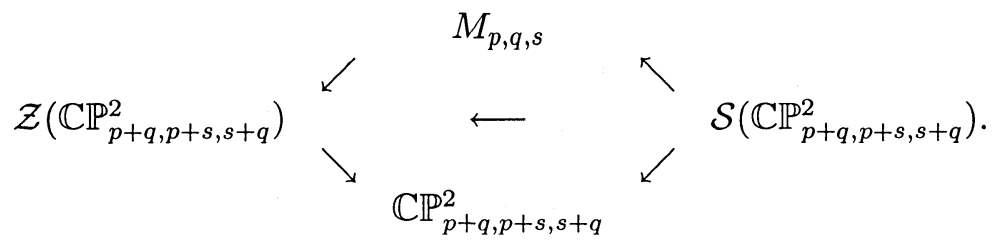

One can view the manifold $S_{p, q, s}$ as a quotient of $N_{p, q, s}=\mu^{-1}(0) \cap S^{11} \subset S^{11} \subset$ $\mathbb{H}^{3} \backslash\{0\}$ by the free circle action (4.22). The manifolds $N_{p, q, s}$ are smooth and compact. For all different values of $(p, q, s) N_{p, q, s}$ is homeomorphic to $S U(3)$. Finally, one can think of $S_{p, q, s}$ as a smooth surface of real codimension 3 in the weighted complex projective space $\mathbb{C P}_{p, p, q, q, s, s}^{5}$. The 3-Sasakian manifold $S_{p, q, s}$ is compact and simply connected. It cannot have constant curvature as it would have to be isometric to the standard 7-sphere. It follows now from the work of Tanno [Ta1] that

$$
\operatorname{dim} \operatorname{Isom}\left(S_{p, q, s}\right)-\operatorname{dim} A u t\left(S_{p, q, s}\right)=2,
$$

where $\operatorname{Aut}\left(S_{p, q, s}\right)$ is the automorphism group of any Sasakian structure of $S_{p, q, s}$. Moreover, the Lie algebra of the Killing vector fields on $S_{p, q, s}$ splits as $\mathfrak{i}\left(S_{p, q, s}\right)=\mathfrak{s p}(1) \oplus \mathfrak{h}$, where $\mathfrak{s p}(1)$ is the Lie algebra of the vector fields generated by the $S p(1)$ action. This implies that $S_{p, q, s}$ cannot be homogeneous unless $p=q=s=1$. We have

Proposition 4.2. The simply connected 3-Sasakian manifold $S_{p, q, s}$ carries an Einstein metrics of positive scalar curvature. $S_{1,1,1}=S U(3) / U(1)$ with a homogenous metric. However, $S_{p, q, s}$ is non-homogeneous for all other values of $(p, q, s)$. 
The existence of a second non-isometric Einstein metric on $S_{p, q, s}$ follows from Theorem 2.5. The self-dual Einstein metric on $\mathbb{C P}_{p+q, p+s, s+q}^{2}$ is a generalization of the standard self-dual positive scalar curvature Einstein metric on $\mathbb{C P}^{2}$. In view of the classification of self-dual Einstein 4-manifolds by Hitchin $[\mathrm{H}]$, any such generalization would involve singularities. In our case $\mathbb{C P}_{p+q, p+s, s+q}^{2}$ has just three isolated orbifold points. In the same spirit $\mathcal{S}\left(\mathbb{C P}_{p+q, p+s, s+q}^{2}\right)$ generalizes the 3-Sasakian structure on $S U(3) / U(1)$ which is an $\mathbb{R} \mathbb{P}^{3}$ bundle over $\mathbb{C P}^{2}$. But now $\mathcal{S}\left(\mathbb{C P}_{p+q, p+s, s+q}^{2}\right)$ is a smooth manifold rather than an orbifold. Note that in the case when $(p+q+s)$ is odd the manifold $S_{p, q, s}$ has a locally free $S O(3)$ action ( $\mathbb{Z} / 2$ subgroup of $S p(1)$ acts trivially). The quotient map $S_{p, q, s} / S O(3)$ gives $\mathbb{C P}_{\frac{p+q}{2}, \frac{q+s}{2}, \frac{p+s}{2}}^{2}$. When $p=q=s=1$ we recover our standard example of $S O(3)$ bundle over $\mathbb{C P}^{2}$.

Wang showed that, if one considers $N=S U(3)$ and

$$
T_{k, l}=\operatorname{diag}\left(\tau^{k}, \tau^{l}, \tau^{-(k+l)}\right)
$$

as its circle subgroup, where $\tau$ is the parameter on the circle and $(k, l)=$ 1 , then the quotient manifold $M_{k, l}=N / T_{k, l}$ admits an $S U(3)$-homogeneous Einstein metric for all such $k$ and $l$. Moreover, as $H^{4}\left(M_{k, l}, \mathbb{Z}\right)$ is the cyclic group of order $\left|k^{2}+l^{2}+k l\right|$, there are infinitely many homotopy types among the $M_{k, l}$ [Wan2]. We would like to point out that our 7 -manifold $S_{p, q, s}$ is homeomorphic to a quotient of $S U(3)$ by a different free circle action. This action can be described as follows: Let $A=\operatorname{diag}\left(\tau^{p}, \tau^{q}, \tau^{s}\right), \Phi \in S U(3) \simeq$ $N_{p, q, s}$, and $B=\operatorname{diag}\left(1,1, \tau^{-(p+q+s)}\right)$. Then $S_{p, q, s}$ is the quotient of $S U(3)$ by the free action

$$
\Phi \stackrel{\tau}{\rightarrow} A \Phi B
$$

This circle action is not a subgroup of $S U(3)$. Our metrics, however, can be computed explicitly. They are non-homogeneous unless $p=q=s=1$, in which case $M_{1,1}=S_{1,1,1}=\mathcal{S}\left(\mathbb{C P}^{2}\right)=S U(3) / U(1)$.

This example readily generalizes to higher dimension. A detailed study of the homotopy types and the diffeomorphism types within a fixed homotopy type as well as the geometry of these and other examples is currently in progress [BGM]. 


\section{EXAMPLE 4.6. OTHER 4-DIMENSIONAL ORBIFOLDS.}

Recently all the Asymptotically Locally Euclidean (ALE) hyperkähler gravitational instantons were obtained as hyperkähler quotients [Kr1, Kr2]. For any discrete subgroup $\Gamma \subset S p(1)$ consider the flat quaternionic vector space $\mathbb{H}^{|\Gamma|}$. There is a unique subgroup $G(|\Gamma|)$ of the unitary group $U(|\Gamma|) / U(1)$ together with a group homomorphism $A: G(|\Gamma|) \rightarrow S p(|\Gamma|)$, defining a hyperkähler action on $\mathbb{H}^{|\Gamma|}$ by

$$
\mathbb{H}^{|\Gamma|} \ni \vec{u} \stackrel{g}{\mapsto} A(g) \vec{u} \quad \text { for } \quad g \in G(|\Gamma|) .
$$

The hyperkähler quotient of $\mathbb{H}|\Gamma|$ by this action gives all the hyperkähler ALE spaces. Choose any homomorphism $b: G(|\Gamma|) \rightarrow S p(1)$. We can define an action of $G(|\Gamma|)$ on $\mathbb{H}^{|\Gamma|+1} \backslash\{0\}$ by

$$
\mathbb{H}^{|\Gamma|+1} \backslash\{0\} \ni(\vec{u}, w) \stackrel{g}{\mapsto}(A(g) \vec{u}, b(g) w) \quad \text { for } \quad g \in G(|\Gamma|) .
$$

Under certain assumptions on the homomorphism $b$ [GN], the hyperkähler quotient of $\mathbb{H}^{|\Gamma|+1} \backslash\{0\}$ by the action above with respect to the zero-level set is an 8-dimensional hyperkähler manifold with an $S O(3)$ action rotating the hyperkähler structure and satisfying the condition of Theorem 1.4. It is the associated bundle of the positive scalar curvature self-dual Einstein orbifolds $\mathcal{O}(\Gamma, b)$ constructed in [GN]. Just as in the previous example, the level sets of the hyperkähler potential function on $\mathcal{U}(\mathcal{O}(\Gamma, b))$ are, in fact, compact smooth 3-Sasakian manifolds. Hence, for any $\Gamma$ and an appropriate choice of $b$ we can construct a compact Einstein 7-manifold $\mathcal{S}(\mathcal{O}(\Gamma, b))$. Let us illustrate this construction with a simple case.

When $\Gamma=\mathbb{Z} / 3$ then $G(\Gamma)=U(1) \times U(1)$. If $g=\left(e^{i s}, e^{i t}\right) \in U(1) \times U(1)$ we have

$$
A(g)=\left(\begin{array}{ccc}
e^{i s} & 0 & 0 \\
0 & e^{i(t-s)} & 0 \\
0 & 0 & e^{-i t}
\end{array}\right) \in S p(3) .
$$

Then Kronheimer's construction gives the following action of $U(1) \times U(1)$ on $\mathbb{H}^{3}$ :

$$
g \cdot\left(u_{1}, u_{2}, u_{3}\right)=\left(e^{i s} u_{1}, e^{i(t-s)} u_{2}, e^{-i t} u_{3}\right)
$$


and the hyperkähler momentum maps is

$$
\mu\left(u_{1}, u_{2}, u_{3}\right)=\left(\begin{array}{c}
\bar{u}_{1} i u_{1}-\bar{u}_{2} i u_{2} \\
\bar{u}_{2} i u_{2}-\bar{u}_{3} i u_{3}
\end{array}\right) \in \mathbb{R}^{2} \otimes \mathfrak{s p}(1) .
$$

Now

$$
\mu^{-1}\left(\begin{array}{l}
\xi_{1} \\
\xi_{2}
\end{array}\right) / U(1) \times U(1)=M(\mathbb{Z} / 3, \xi),
$$

where $\xi \in \mathbb{R}^{2} \otimes \mathfrak{s p}(1)$ is chosen in such a way that the $G(\Gamma)$ action on the level set of the moment map is free, is the two-center multi-Eguchi-Hanson metric. It is easy to see that it is enough to assume that $\xi_{1} \neq-\xi_{2}$. Now we consider a homomorphism $b: U(1) \times U(1) \rightarrow S p(1)$

$$
b\left(e^{i s}, e^{i t}\right)=e^{i a s+i b t} \in S p(1), \quad a, b \in \mathbb{Z} .
$$

so that the action of $U(1) \times U(1)$ extends to $\mathbb{H P}^{3} \ni\left[u_{0}, u_{1}, u_{2}, u_{3}\right]$ as

$$
g \cdot\left[u_{0}, u_{1}, u_{2}, u_{3}\right]=\left[e^{i(a s+b t)} u_{0}, e^{i s} u_{1}, e^{i(t-s)} u_{2}, e^{-i t} u_{3}\right]
$$

and the quaternionic Kähler moment map is

$$
\tilde{\mu}\left(\left[u_{0}, u_{1}, u_{2}, u_{3}\right]\right)=\left(\begin{array}{c}
\bar{u}_{1} i u_{1}-\bar{u}_{2} i u_{2}+a \bar{u}_{0} i u_{0} \\
\bar{u}_{2} i u_{2}-\bar{u}_{3} i u_{3}+b \bar{u}_{0} i u_{0}
\end{array}\right) .
$$

Consider the $S p(1)$-invariant zero section $\mu^{-1}\left(\begin{array}{l}0 \\ 0\end{array}\right)$ in $\mathbb{H P}^{3}$. For any $a, b \in \mathbb{Z} \backslash\{0\}$, $a \neq-b$ the action of $U(1) \times U(1)$ is locally free on $\mu^{-1}\left(\begin{array}{l}0 \\ 0\end{array}\right) \in \mathbb{H}^{3}$. Hence

$$
\mathcal{O}\left(\mathbb{Z}_{3} ; a, b\right)=\mu^{-1}\left(\begin{array}{l}
0 \\
0
\end{array}\right) / U(1) \times U(1)
$$

is a compact 4-dimensional orbifold with self-dual Einstein metric and positive scalar curvature.

Let us examine the lift of the above quotient to the associated bundle of $\mathbb{H}^{3}$. There we have the hyperkähler momentum map

$$
\mu\left(u_{0}, u_{1}, u_{2}, u_{3}\right)=\left(\begin{array}{l}
\bar{u}_{1} i u_{1}-\bar{u}_{2} i u_{2}+a \bar{u}_{0} i u_{0} \\
\bar{u}_{2} i u_{2}-\bar{u}_{3} i u_{3}+b \bar{u}_{0} i u_{0}
\end{array}\right) \in \mathbb{R}^{2} \otimes \mathfrak{s p}(1)
$$

It is easy to see that the action of $U(1) \times U(1)$ is, in fact, free on $\mu^{-1}\left(\begin{array}{l}0 \\ 0\end{array}\right) \in$ $\mathbb{H}^{4} \backslash\{0\}$ provided $a \neq-b, a, b \in \mathbb{Z} \backslash\{0\}$. It is, therefore, free on $N(\mathbb{Z} / 3 ; a, b)=$ 
$\mu^{-1}\left(\begin{array}{l}0 \\ 0\end{array}\right) \cap \nu^{-1}(c) \subset \mathbb{H}^{4} \backslash\{0\}$, where

$$
\nu\left(u_{0}, u_{1}, u_{2}, u_{3}\right)=\left(\bar{u}_{0} u_{0}+\bar{u}_{1} u_{1}+\bar{u}_{2} u_{2}+\bar{u}_{3} u_{3}\right) .
$$

The manifold $N(\mathbb{Z} / 3 ; a, b)$ is a compact surface in $\mathbb{H}^{4} \backslash\{0\}$ of real codimension 7. The quotient of $N(\mathbb{Z} / 3 ; a, b)$ by $U(1) \times U(1)$ is a compact 7-manifold for any nonzero integers $a$ and $b$ where $a \neq-b$ and it carries a 3-Sasakian structure. By construction

$$
N(\mathbb{Z} / 3 ; a, b) / U(1) \times U(1)=\mathcal{S}(\mathcal{O}(\mathbb{Z} / 3 ; a, b))
$$

and

$$
\mathcal{S}(\mathcal{O}(\mathbb{Z} / 3 ; a, b)) / S O(3)=\mathcal{O}(\mathbb{Z} / 3 ; a, b)
$$

Let us remark that in the case of $\Gamma=\mathbb{Z} / k$, the group $G(\Gamma)=T^{k-1}$. Any homomorphism $b: T^{k-1} \rightarrow S p(1)$ can be written as

$$
T^{k-1} \ni\left(e^{2 \pi i t_{1}}, \ldots, e^{2 \pi i t_{k-1}}\right) \mapsto e^{2 \pi i\left(\alpha_{1} t_{1}+\cdots+\alpha_{k-1} t_{k-1}\right)} \in S p(1)
$$

where $\left(\alpha_{1}, \ldots, \alpha_{k-1}\right) \in \mathbb{Z}^{k-1}$. In this case, using our construction, we obtain the 3-Sasakian 7-manifold

$$
S_{\alpha_{1}, \ldots, \alpha_{k-1}}=\mathcal{S}\left(\mathcal{O}\left(\mathbb{Z} / k ; \alpha_{1}, \ldots, \alpha_{k-1}\right)\right)
$$

involving $k-1$ integers $\left(\alpha_{1}, \ldots, \alpha_{k-1}\right)$ such that $\sum_{k=1}^{l} \alpha_{l} \neq 0$ for all $1 \leq l \leq$ $k-1$.

EXAMPle 4.7. The MOdUli SPACE OF InSTANTONS.

The moduli spaces of instantons (self-dual connections with respect to a conformal class of metrics) associated to principal $G$ bundles over Riemannian four-manifolds give another class of examples of hyperkähler manifolds with quaternionic Kähler reductions. It had been observed by Donaldson, Hitchin, Taubes, and others that the self duality equations on $S^{4}$ have the interpretation of an infinite dimensional hyperkähler moment map with respect to the action of the based gauge group on the infinite dimensional affine space of $G$ connections. Hence, the hyperkähler quotient procedure of [HKLR] gives the moduli space, which we denote by $\mathcal{M}_{k}(G)$ the structure of a hyperkähler manifold. Hitchin also observed that the quadratic constraints of 
the well-known ADHM construction [ADHM] can be interpreted as the zero set of a hyperkähler moment map with respect to the natural $O(k)$ action that occurs there. Furthermore, it has now been proven by Kronheimer and Nakajima $[\mathrm{KrNa}]$ and by Maciocia $[\mathrm{M}]$ that these two methods give equivalent hyperkähler structures on $\mathcal{M}_{k}(G)$.

A detailed study of the hyperkähler geometry on $\mathcal{M}_{k}(G)$, obtained by hyperkähler reduction from the ADHM construction is given in [BoMa1]. There it is shown that the hyperkähler geometry of the ADHM construction is a special case of a more general theory that is relevant to the discussion in Section 1. Let $\mathfrak{g}$ denote a real orthogonal symmetric Lie algebra. The tensor product over the reals $\mathfrak{g} \otimes \mathbb{H}$ has a natural algebraic structure determined by the Lie algebra on $\mathfrak{g}$ and the quaternionic algebra structure of $\mathbb{H}$. In [BoMa1] this algebraic structure is called the quaternionic adjoint map. If $\mathfrak{p}$ denotes the plus one eigenspace in the symmetric space decomposition of $\mathfrak{g}$, then the diagonal restriction of the quaternionic adjoint map is a hyperkähler moment map on $\mathfrak{p} \otimes \mathbb{H}$ with respect to the adjoint action of the Lie group $K$ generated by the minus one eigenspace of $\mathfrak{g}$. The ADHM construction is associated to the $K$ module $\mathfrak{p}(\mathbb{H})$ obtained as the direct sum of the plus one eigenspaces of the symmetric Lie algebras $\mathfrak{g} l(k, \mathbb{R})$ and $n$ copies of $\mathfrak{o}(k, 1)$ tensored with $\mathbb{H}$.

For example, we have

Proposition 4.3 ([BoMa1, 4.5.3]). $\mathcal{M}_{k}$ has a projectivization $\mathbb{P M}_{k}$. Moreover, for $k>1$, there is a principal fibration $\mathbb{H}^{*} \rightarrow \mathcal{M}_{k} \rightarrow \mathbb{P} \mathcal{M}_{k}$ such that the quotient manifold $\mathbb{P M}_{k}$ is a quaternionic Kähler manifold.

This proposition implies that $\mathcal{M}_{k}$ is an example of an associated hyperkähler bundle to the quaternionic Kähler manifold $\mathbb{P} \mathcal{M}_{k}$. Moreover, the obstructions of Section 1 vanish, and the whole diagram (0.3) applies. While the original motivation in [BoMa1] was to study the topology of $\mathcal{M}_{k}$, the techniques and results in the first half of that paper are closely related to some of the ideas and constructions in this paper. Hence, the reader is referred to the first half of [BoMa1] to see further connections between instantons and the results of this paper. 


\section{REFERENCES}

[A] Alekseevskii, D. V., Classification of quaternionic spaces with solvable group of motions, Math. USSR-Izv., 9 (1975), 297-339.

[ADHM] Atiyah, M.F., Drinfeld, V.G., Hitchin, N.J. and Manin, Y.I., Construction of instantons, Phys. Lett. A, 65 (1978), 185-187.

[AHS] Atiyah, M.F., Hitchin N.J. and Singer, I., Self-duality in four dimensional Riemannian geometry, Proc. Roy. Soc. London, Ser. A, 362 (1978), 425-461.

[BéBer] Bérard Bergery, L., Sur de nouvelles variétiés riemanniennes d'Einstein, Publications de l'Institut E. Cartan nô 4 (Nancy) (1982), 1-60.

[Ber] Berger, M., Sur les groupes d'holonomie des variètés à conexion affines et des variètés riemanniennes, Bull. Soc. Math. France, 83 (1955), 279-330.

[Bes] Besse, A.L., Einstein manifolds, Springer-Verlag, New York 1987.

[BGM] Boyer, C.P., Galicki, K. and Mann, B.M., Einstein metrics and singular fibrations over quaternionic Kähler and Kähler-Einstein orbifolds, in preparation.

[BoMa1] Boyer, C.P. and Mann, B.M., The hyperkähler geometry of the ADHM construction and quaternionic geometric invariant theory, Proc. of Sym. in Pure Math.: Differential Geometry, Amer. Math. Soc., 54 (1993) Part 2.

[BoMa2] Boyer, C.P. and Mann, B.M. , Instantons, quaternionic Lie algebras, and hyperkähler geometry, Proc. Ann. Sem. Can. Math. Soc. on Lie Theory, Differential Equations and Representation Theory, Univ. de Montreal Press (1990), 119-136.

[CSal] Capria, M. M. and Salomon, M. S., Yang-Mills fields on quaternionic spaces, Nonlinearity 1 (1988), 517-530.

[F] Futaki, A., Kähler-Einstein Metrics and Integral Invariants, Lecture Notes in Mathematics vol. 1314, Springer-Verlag 1988.

[G1] Galicki, K., Multi-centre metrics with negative cosmological constant, Class. Quantum Grav., 8 (1991), 1529-1543.

[G2] Galicki, K., Generalization of the momentum mapping construction for quaternionic Kähler manifolds, Commun. Math. Phys., 108 (1987), 117138.

[G3] Galicki, K., Geometry of the scalar couplings in $N=2$ supergravity models, Class. Quantum Grav., 9 (1992), 27-40.

[GL] Galicki, K. and Lawson, B.H. Jr., Quaternionic reduction and quaternionic orbifolds, Math. Ann., 282 (1988), 1-21.

[GN] Galicki, K. and Nitta, T., Non-zero scalar curvature generalizations of the ALE hyperkähler metrics, J. Math. Phys., 33 (1992), 1765-1771.

[GP] Galicki, K. and Poon, Y. S., Duality and Yang-Mills Fields on Quaternionic Kähler Manifolds, J. Math. Phys. 33 (1991), 1263-1268.

[H] Hitchin, N.J,. Kählerian twistor spaces, Proc. Lond. Math. Soc., 43 (1981), 133-150.

[HKLR] Hitchin, N.J., Karlhede, A., Lindström, U. and Roček, M., Hyperkähler metrics and supersymmetry, Comm. Math. Phys., 108 (1987), 535-589.

[I1] Ishihara, S., Quaternion Kählerian manifolds, J. Diff. Geom., 9 (1974), 483- 
500.

[I2] Ishihara, S., Quaternion Kählerian manifolds and fibered Riemannian spaces with Sasakian 3-structure, Kodai Math. Sem. Rep., 25 (1973), 321-329.

[IK] Ishihara, S. and Konishi, M., Fibered Riemannian spaces with Sasakian 3structure, Differential Geometry, in honor of K. Yano, Kinokuniya, Tokyo (1972), 179-194.

[Ka] Kashiwada, T. , A note on a Riemannian space with Sasakian 3-structure, Nat. Sci. Reps. Ochanomizu Univ., 22 (1971), 1-2.

[KoiSa1] Koiso, N. and Sakane, Y., Curvature and topology of Riemannian manifolds, Katata (1985), 165-179, Lecture Notes in Math. vol. 1201, Springer, BerlinNew York 1986.

[KoiSa2] Koiso, N. and Sakane, Y., Nonhomogeneous Kähler-Einstein metrics on compact complex manifolds II, Osaka J. Math., 25 (1988), 933-959.

[Kon] Konishi, M. , On manifolds with Sasakian 3-structure over quaternion Kählerian manifolds, Kodai Math. Sem. Reps., 26 (1975), 194-200.

[Kr1] Kronheimer, P. B., The construction of ALE spaces as hyperkähler quotients, J. Diff. Geom., 29 (1989), 665-683.

[Kr2] Kronheimer, P. B., A Torelli-type theorem for gravitational instantons, J. Diff. Geom., 29 (1989), 685-697.

[Kr3] Kronheimer, P. B., Instantons and the geometry of the nilpotent variety, J. Diff. Geom., 32 (1990), 473-490.

[KrNa] Kronheimer, P. B. and Nakajima, H., Yang-Mills instantons on ALE gravitational instantons, preprint (1990) Oxford.

[Ku] Kuo, Y.-Y., On almost contact 3-structure, Tôhoku Math. J., 22 (1970), 325-332.

[L] LeBrun, C., Quaternionic-Kähler manifolds and conformal geometry, Math. Ann., 284 (1989), 353-376.

[M] Maciocia, A., Metrics on the moduli space of instantons over Euclidean 4sphere, Commun. Math. Phys., 135 (1991), 467-482.

[MR] Marchiafava, S. and Romani, G., Sui fibrati con struttura quaternioniale generalizzata, Ann. Mat. Pura Appl. 107 (1976), 131-157.

[Mo] Molino, P., Riemannian Foliations, Birkhäuser, Boston 1988.

[OW] Orlik, P., and Wagreich, P., Seifert n-manifolds, Invent. Math., 28 (1975), 137-159.

[Pa] Page, D., A compact rotating gravitational instanton, Phys. Lett., 79B (1979), 235-238.

[Pe] Penrose, R., Nonlinear gravitons and curved twistor theory, Gen. Relativ. Grav.,1 (1976), 31-52.

[PePo1] Pedersen, H. and Poon, Y.S., Hamiltonian constructions of Kähler-Einstein metrics and Kähler metrics of constant scalar curvature, Commun. Math. Phys., 136 (1991), 309-326.

[PePo2] Pedersen, H. and Poon, Y.S., Twistorial construction of quaternionic manifolds, Proc. VIth Int. Coll. on Diff. Geom., Cursos y Congresos, Univ. Santiago de Compostela, 61 (1989), 207-218.

[PoSal] Poon, Y. S. and Salamon, S,. Eight-dimensional quaternionic Kähler mani- 
folds with positive scalar curvature, J. Diff. Geom., 33 (1990), 363-378.

[Sal1] Salamon, S., Quaternionic Kähler manifolds, Invent. Math., 67 (1982), 143171.

[Sal2] Salamon, S., Differential geometry of quaternionic manifolds, Ann. Sci. Ec. Norm. Sup. Paris, 19 (1986), 31-55.

[Sas1] Sasaki, S., Spherical space forms with normal contact metric 3-structure, J. Diff. Geom., 6 (1972), 307-315.

[Sas2] Sasaki, S., On differentiable manifolds with certain structures which are closely related to almost contact structure, Tôhoku Math. J., (2) 12 (1960), 459-476.

[SasHa] Sasaki, S. and Hatakeyama, Y., On differentiable manifolds with contact metric structure, J. Math. Soc. Japan, 14 (1962), 257-279.

[Sw1] Swann, A. F., Hyperkähler and quaternionic Kähler geometry, Math. Ann., 289 (1991), 421-450.

[Sw2] Swann, A. F., Hyperkähler and quaternionic Kähler geometry, Ph. D. Thesis, Oxford 1990.

[Ta1] Tanno, S., On the isometry of Sasakian manifolds, J. Math. Soc. Japan, 22 (1970), 579-590.

[Ta2] Tanno, S., Killing vectors on contact Riemannian manifolds and fiberings related to the Hopf fibrations, Tôhoku Math. J. 23 (1971), 313-333.

[Th] Thomas, C. B., Almost regular contact manifolds, J. Diff. Geom., 11 (1976), 521-533.

[TY] Tain, G. and Yau, S. T., Kähler-Einstein matrics on complex surfaces with $c_{1}>0$, Comm. Math. Phy. 112 (1987), 175-203.

[Wan1] Wang, M., Einstein metrics and quaternionic Kähler manifolds, Math. Z., 210 (1992), 305-326.

[Wan2] Wang, M., Some examples of homogeneous Einstein manifolds in dimension seven, Duke Math. J., 49 (1982), 23-28.

[WanZi1] Wang, M. and Ziller, W., Curvature and topology of Riemannian manifolds, Katata (1985), 319-338, Lecture Notes in Math. vol. 1201, Springer, BerlinNew York 1986.

[WanZi2] Wang, M. and Ziller, W., Einstein metrics on principal torus bundles, J. Diff. Geom., 31 (1990), 215-248.

[Wo] Wolf, J. A., Complex homogeneous contact manifolds and quaternionic symmetric spaces, J. Math. Mech., 14 (1965), 1033-1047.

[Ya] Yano, K., Integral Formulas in Riemannian Geometry, Marcel Dekker, Inc., New York 1970.

[YK] Yano, K. and Kon, M., Structure on Manifolds, World Scientific Press, 1984.

University of New MeXiCo, U. S. A.

E-mail address: cboyer@math.unm.edu, galicki@math.unm.edu, mann@math.unm.edu

RECEIVED OCTOBER 8, 1992. 